\title{
Abstracts
}

\section{Evidence-Based Complementary Medicine}

State of the Evidence $\cdot$ Methodological Challenges

Congress Presidents

International Congress on Clinical Research and

D. Melchart, Munich Quality Management in Complementary Medicine

D. Eisenberg, Boston, MA

Munich, April 6 to 8, 2000

Organized by

The Centre for Complementary Medicine Research Dept. Internal Medicine II, Technische Universität Munich

in Collaboration with

The Center for Alternative Medicine Research and Education, Beth Israel Deaconess Medical Center, Harvard Medical School

Sponsored by Erich Rothenfußer Stiftung, Munich 
A total of 80 abstracts have been submitted for presentation on the EBCM Congress. The abstracts came from 14 different countries, the majority from Europe, followed by USA and Canada. The following table gives the most frequent topics.

Topics of submitted abstracts $(n=80)$

Acupuncture 22

Classical naturopathic methods $\quad 15$

Phytotherapy 14

Homeopathy 9

Various themes 20

In order to ensure a high quality level and to take into account the limited capacity of the schedule as well, the Scientific Committee of the congress performed a selection process. The abstracts were evaluted by 15 experts. Each abstract was seen by 2 reviewers. The abstracts were presented as blind copy, i. e. without the authors' names, affiliations or addresses. A total of 65 (81.25\%) abstracts were finally selected for presentation. The section on pages 38-57 lists these abstracts in alphabetical order. 30 abstracts were selected for oral presentation and 35 were selected for poster presentation.

In addition to these peer-reviewed abstracts, you find the abstracts of the invited speakers on pages 31-37.

We gratefully thank all reviewers and all members of the Organizing Committee involved in the process of abstract evaluation. 


\section{Abstracts Invited Speakers}

Prevalence Patterns of Complementary and Alternative Medical (CAM) Use: Implications for the Future

Eisenberg, $D$.

Harvard Medical School, Boston, MA, USA

Patterns of complementary and alternative medical (CAM) therapy use in the United States and highlight comparable survey data from the United Kingdom and Europe will be summarized. New analyses of U.S. data suggest that the increased popularity of CAM therapy use is not a 'fad' but rather a trend which has been in progress for at least the past seven decades. Other analyses suggest that CAM therapy use is not limited to younger adults but also is used by more than $30 \%$ of adults aged 65 or older. In light of these data, it can be argued that patients will continue to seek advice about the responsible use or avoidance of CAM therapies well into the next century.

Given these data, it can be argued that the international biomedical community would be wise to share challenges inherent in developing models of 'integrative' medical care. These include:

1) Perceptions impeding full disclosure and communication between patients and conventional caregivers pertaining to CAM therapies; 2) The need for comparable data collection tools; 3) Methodologic challenges pertaining to CAM study design; 4) The necessity to further explore placebo-related phenomena and the impact of study design on outcome; 5) International standardization and quality assurance of botanical products, vitamins, minerals and other dietary supplements; 6) The necessity for international post-market surveillance of dietary supplements and their interactions with prescription medications and over-the-counter drugs; 7) The creation of inclusion and exclusion criteria for formularies in conventional medical settings; 8) A reasonable approach to quality assurance (i.e., credentialing for providers) of CAM providers; 9) A shared commitment to improve education and training for physicians, nurses, pharmacists and allied health professionals regarding the use or avoidance of CAM therapies.

Taking into consideration our shared appreciation for evidence-based approaches towards disease management and prevention, the abovementioned themes provide a useful backdrop to this scientific symposium.

\section{Can Complementary Medicine Be Evidence-Based?}

\section{Mulrow, C.D.}

Audie Murphy Veterans Affairs Hospital and University of Texas Health Science Center, San Antonio, TX, USA

In the late 20th century, 'evidence-based' medicine was defined as the conscientious, explicit and judicious use of current best evidence in making decisions about the care of individual patients. Practicing such 'evidence-based' health care purportedly required integration of professional expertise, patient preference, and external scientific evidence. It neither required nor emphasized ignoring culture, beliefs, history, experience, or biological evidence. Rather, it emphasized incorporation of clinically relevant scientific evidence into health care decision-making. Clinically relevant scientific evidence was regarded as information derived from well-designed studies in humans that minimized patient and professional bias.

Can Complementary Medicine be 'evidence-based'?
Complementary medicine is an incredibly diverse field. It encompasses ancient practices as well as innovations that are new to the 21st century; multiple theories and schools; and myriad biological, physical, mental, and spiritual therapies. Becoming 'evidence-based' will require a critical attitude and a willingness to replace old theories and practices with new. It will require acceptance of the fallibility of history and experience, and continual learning based on scientific experiments. For some, it will require building a body of credible scientific evidence using already established experimental methodologies. For others, it will require development and application of new research techniques. For all, it will require an appreciation of clinical research with humans.

Realization of 'evidence-based' complementary medicine will not require reducing patients to biological organisms and their illnesses or lack of health to purely physiochemical phenomena. It will not rely on betrayal of culture and experience, but rather on a philosophical evolution that incorporates and values highly human scientific evidence. Thus, the answer: complementary medicine can be evidence-based if that is what the field wants and is willing to do.

\section{Evidence-Based Art (of Medicine)}

Reilly, D.

Glasgow Homoeopathic Hospital \& Glasgow University, UK

Just as a painter wants true colours and good brushes, but sometimes must make do with a bad situation and materials, so too in the Art of Medicine. We want sharp and appropriate tools in our tool box, be they herbs, or scalpels - and RCTs can sometimes help by identifying the best fitting specific tools - but then we must work and shape the actual encounter and care with all its complexity, humanity and individuality, using ingenuity and creativity as much as things or therapies. Evidence must rise to this complex beauty. It would be a mistake to judge Michelangelo's David by randomised trials of the tools used to shape it. This is no excuse for bad tools, just a call for the courage to have evidence tackle more than the wee bits of so-called specific effects of particular tools that characterise only a minority of clinical care.

So do our paintings work for our clients? Can they be tested blind? Often the colours are true, the brush strokes precise, but the feel is all wrong. Much dissatisfaction with Western Medicine today is about that feel, not the well-tested pigments. Take an archetypal medical colour: Valium. Is it evidence-based? It 'worked' in placebo-controlled trials, but did it 'work' for us as individuals and as a culture? As the most popular drug on planet earth by the mid 1980s - did it fulfill its promise, was the evidence right?

What sort of care do we want? And do we have 'evidence-based' evidence tools, proven to guide us towards such care? Will such tools be validated in the flame of everyday complexity and care, where the 'non-specific' effects may be more important than the 'specific' and clients have multiple interacting problems, fears and hopes, and our care, kindness and encouragement may be more effective in the long run than Valium. The evidence mosaic needs to be built, and proven, over time.

Take Gordon asking for help after the care of the Pain Relief Clinic, Rheumatologists Neurologist and Orthodpaedic Surgeons. Numerous, expensive and risky 'proven' specific interventions for his chronic neuropathic pain had failed. Yet a good therapeutic encounter, expression of hidden feelings and the use of gentle interventions (massage, acupuncture and homoeopathy) and in 3 days he was transformed - still evident 5 months later - with his suffering gone. Unless our search for evidence is grounded in, and tested against, such complex predicaments - we may miss the picture, the image of healing, while analysing the pigment. 


\section{Under the Metascope: Potentials and Limitations of Systematic Reviews and Meta-Analyses}

Egger, $M$.

MRC Health Services Research Collaboration, Dept. of Social Medicine, University of Bristol, UK

Critical appraisal, systematic review and statistical synthesis of the evidence that is available on the benefits and risks of interventions are important components of any strand of evidence-based medicine, including evidence-based complementary medicine. Using examples from homoeopathy and other interventions from complementary and conventional medicine I will attempt to illustrate the potentials and limitations of the meta-analytical approach and discuss the problems of applying meta-analytical results to the individual patient.

Since Mulrow [1] and Oxman [2] drew attention to the poor quality of narrative reviews it has become clear that conventional reviews are an unreliable source of information. The classical review article tends to reflect the personal opinion of the author and is therefore prone to bias and error. Systematic reviews are characterised by clearly defined objectives and a reproducible methodology that aims to minimise subjectivity and bias [3]. If appropriate and feasible, individual studies are combined statistically in a meta-analysis, a procedure that will enhance the precision of estimates of treatment effects. Meta-analyses are, however, also liable to bias. Several examples exist of meta-analyses of small trials that demonstrated beneficial effects of an intervention (e.g. infusion of magnesium salts in acute myocardial infarction) which large definite trials later failed to confirm (e. g. ISIS-4 trial). The occurrence of misleading meta-analyses is not surprising considering the selective publication of 'positive' findings and the often inadequate quality of component studies. Misleading meta-analyses may also result from the inappropriate combination of heterogeneous studies.

Every patient is unique regarding the exact localisation and severity of the lesion, pre-existing abilities and co-morbidities, social environment, personality and emotional response. The appropriate application of meta-analytic findings to the individual patient is difficult. It requires consideration of both quantitative and qualitative approaches to decision making. Empathic integration of these approaches distinguish the experienced, scientifically and socially competent physician.

\section{References:}

1 Mulrow CD: The medical review article: State of the science. Ann Intern Med 1987:106:485-488.

2 Oxman AD, Guyatt GH: Guidelines for reading literature reviews. Can Med Ass J 1988;138:697-703.

3 Egger M, Smith GD, Altman DG (eds): Systematic Reviews in Healthcare: Meta-Analysis in Context. London, BMJ Books 2000 (in press)

\section{A Systematic Review of Systematic Reviews of Complementary Therapies}

Linde, $K{ }^{1,2}$; ter Riet, G. $^{3}$; Hondras, $M{ }^{4}$; Vickers, $A .^{5}$; Thormählen, ${ }^{1}{ }^{1}$; Melchart, $D .^{1}$

${ }^{1}$ Technische Universität, München; ${ }^{2}$ Humboldt University, Berlin, Germany; ${ }^{3}$ University of Maastricht, The Netherlands; ${ }^{4}$ Consortial Center for Chiropractic Research; ${ }^{5}$ Memorial Sloan-Kettering Cancer Center, New York, NY, USA

Background and Objective: In past years an increasing number of systematic reviews on complementary therapies have been published. We aimed to collect and critically assess these reviews for the Cochrane Collaboration's Complementary Medicine Field. Methods: A variety of databases (Medline, Embase, Cochrane Controlled Trials Registry etc.) and other sources (screening bibliographies, contacting researchers) were searched to identify potentially eligible reviews. To be included reviews had to meet the following inclusion criteria: a) focus on clinical trials on one of 30 predefined complementary therapies; b) focus on treatment effects; c) explicit description of review methods; d) published report available. Two reviewers independently extracted information on characteristics, methods, and results of the included reviews. Scientific quality was assessed using a scale proposed by Oxman and Guyatt. Preliminary Results (December 1999): Over 400 potential systematic reviews were identified of which 238 were selected after excluding papers which clearly did not meet inclusion criteria. More than half of the reviews identified were on either herbal remedies, acupuncture, homeopathy, or manual therapies (chiropractic \& osteopathy). The methodological approaches can be classified into 4 categories: reviews with a strong focus on assessment of methodological quality (most frequently used in older reviews on herbs, acupuncture and homeopathy), quantitative meta-analyses without in-depth account of quality (in diverse areas), pragmatic quantitative reviews investigating factors which influence the results of treatment (frequently in reviews on cognitive therapies), and reviews with both assessment of quality and quantitative summary estimates (mainly in more recent and Cochrane reviews). Final results will be presented at the conference. Preliminary Conclusions: A large number of systematic reviews exists on a variety of complementary therapies. The review methods vary considerably. High-quality reviews have supported the value of at least some complementary therapy interventions.

\section{Placebos and Placebo Effects in Clinical Trials}

\section{Kleijnen, $J$.}

NHS Centre for Reviews and Dissemination, University of York, UK

For many years it has been acknowledged that context factors may considerably influence the size of a therapeutic effect. These extraneous factors, which include placebos, are widely recognised in medicine, but have generally been regarded as a nuisance variable in research.

In order to investigate possible interaction between specific and context factors, a model design was developed, the balanced placebo design. The model is a $2 \times 2$ factorial design, consisting of a pharmacological intervention (the administration of medication or placebo) and a context intervention (e.g. a positive attitude towards the expected therapeutic effect or a neutral attitude towards the expected therapeutic effect).

In a systematic review, we evaluated studies that investigated the influence of a context intervention on the magnitude of the specific effect by using the balanced placebo design. For example, the difference in response between the control and experimental group was smaller if both groups were treated in a supportive atmosphere, than if both groups were treated in a neutral environment [1].

In another review, we assessed the impact of colour on perceived effect and effect size of medication and we examined whether antidepressant medication available in the Netherlands is differently coloured compared to hypnotic, sedative and anxiolytic medication [2].

In a third review, we assessed whether subcutaneous placebo administration is associated with an enhanced placebo effect compared with oral administration. Furthermore, we investigated whether placebo administration within hospital is associated with an enhanced placebo effect compared with placebo treatment at home [3].

In a fourth review, we assessed whether frequency of placebo administration is associated with duodenal ulcer healing [4]. 
In a randomised clinical trial we aimed to investigate whether experimentally induced expectancy can modify the analgesic effect of tramadol relative to placebo in chronic pain patients [5].

In the presentation, the findings of these studies and their implications will be discussed.

\section{References:}

1 Kleijnen J, de Craen AJM, van Everdingen J, Krol L: Placebo effect in double blind clinical trials: A review of interactions with medications. Lancet 1994;344:1347-1349.

2 De Craen AJM, Roos PJ, de Vries AL, Kleijnen J: Effects of coloured medication. Br Med J 1996;313:1624-1626.

3 De Craen AJM, Moerman DE, Heisterkamp SH, Tytgat GNJ, Tijssen JGP, Kleijnen, J: Placebo effect in the treatment of duodenal ulcer: The more placebos per day, the better the healing. Br J Clin Pharmacol 2000 (in press).

4 De Craen AJM, Tijssen JGP, De Gans J, Kleijnen J: Placebo effect in the acute treatment of migraine: Subcutaneous placebos are better than oral placebos. J Neurol 2000 (in press).

5 De Craen AJM: Placebos and placebo effects in clinical trials. Thesis, University of Amsterdam, 1998. ISBN 90-9011864-0.

\section{The Placebo in the Evaluation of Complementary Medicine}

Kaptchuk, T.J.

Center for Alternative Medicine Research and Education, Harvard Medical School, Boston, MA, USA

The following issues will be discussed in the presentation:

A. Historical Issues

- The development of placebo controls in scientific evaluation as the conventional response to unconventional medical claims [1].

- The placebo's role in the rhetorical debate between conventional and unconventional medicine [2].

B. Implementation Issues

- Difficulties with designing placebo controls: acupuncture, manual therapy, complex interventions, psychotherapy [3].

- Unanticipated large placebo effect.*

C. Methodological Issues

- Effects of 'double blinding' versus 'single blinding' on outcomes in RCTs [4].

- Outcomes of RCTs are effected by whether comparison is with placebo or another form of treatment [5].

- Effects of being in an RCT [6].

D. Philosophical and Ethical Issues

Veracity versus efficacy: does the placebo-controlled double-blind RCT produce a scientific judgment or an ethical judgment? [7]

\section{References:}

1 Kaptchuk TJ: Intentional ignorance: A history of blind assessment and placebo controls in medicine. Bull Hist Med 1998;72:389-433.

2 Kaptchuk TJ: Powerful placebo: The dark side of the randomized controlled trial. Lancet 1998;351:1722-1725.

3 Vincent CA, Lewith G: Placebo controls for acupuncture studies. J Roy Soc Med 1995;88:199-202.

4 Gracely RH. et al: Clinicians' expectations influence placebo analgesia. Lancet 1985;i:43.

5 Rochon PA. et al: Are randomized controlled trials outcomes influenced by the inclusion of a placebo group? A systematic review of nonsteroidal anti-inflammatory drug trials for arthritis treatment? J Clin Epidemiol 1999;52:113-122.

6 Bergmann J. et al: A randomised clinical trial of the effecct of informed consent on the analgesic activity of placebo and Naproxen in cancer pain. Clin Trials Meta-Anal 1994;29:41-47.

7 Sullivan MD: Placebo controls and epistemic control in orthodox medicine. J Med Philos 1993;18:213-231.

* Kaptchuk TJ: Do devices have enhanced placebo effects? J Clin Epid 2000 (in press).

\section{What Patients Expect from Complementary Medicine Research}

Harrer, $B$.

Patient Information Center for Natural Medicine, Berlin, Germany

Objectives: What do patients expect and demand from complementary medicine research? What evidence is wanted? Methods: (1) Since 1995 the Patient Information Center for Natural Medicine documents all its consultations. About 1,000 phonecalls and 600 E-mails per year are registered. (2) In 1999, additionally the quality of the consultations was researched. Patients were interviewed some days after the consultation using a questionnaire. (3) Other projects for patient information and self-help groups were interviewed. In 1999 many health information projects in Berlin gathered into the "Berlin Forum for Patients' Interests". Patients' demands for a humane medicine are discussed in a working group. Results: Patients eighter ask for complementary therapies to use them in addition to orthodox treatment or they ask for alternative methods instead of orthodox medicine with high risks and side effects. Chronic patients are often interested in new research and clinical studies but also follow advices of other patients or therapists whose practical experiences show positive results. Patients are willing to try out new hopeful methods only if they have been introduced to the study in an open and honest manner. Patients' most important demands are:

- honest therapists who respect the patient's will,

- holistic understanding,

- understandable information about sickness and therapy, possibilities and risks,

- all therapies should be paid by health insurances,

- better continuing education of therapists using the wealth of experience of self-help groups,

- protection against frauds and against being abused by medical research.

Conclusion: Patients demand that research shows the pros and cons of therapies and they want open access to such research papers. On the other hand many patients say that the personality of the therapist is more important than the methods used. They are willing to trust the therapist's experience and do not care whether the suggested therapies are tested in controlled trials, as long as these therapies are safe and have no adverse effects.

\section{A Provider's Perspective: Current Issues in Providing and Funding Complementary Medical Care}

Lewith, G.T.

University of Southampton, UK

Complementary and alternative medicine (CAM) use has increased dramatically over the last decade. The issues we need to address as providers are:

- Why do people seek CAM?

- Is it being provided in an appropriate manner?

- How should it be delivered?

- Is it cost-effective?

Individuals who seek CAM tend to do so because of the desire to manage their frequently chronic health problems in an egalitarian manner and in the context of a medical model with which they feel comfortable. These observations should encourage us to reflect on what patients need from us as healthcare practitioners. A variety of models for CAM provision exist within Europe and the United States; all have a number of advantages and disadvantages that will be discussed. No ideal model for CAM provision currently exists. Clinical governance, quality control and clinical audit must begin to form an essential part of CAM de- 
livery. This will involve professional cooperation as an essential step in the delivery of affordable, accessible, acceptable and efficient CAM services. The hierarchy of evidence for CAM must be thoughtfully structured and developed, and must necessarily involve case studies and outcomes research, as well as randomised controlled trials. This can only be achieved within a sympathetic and cooperative research environment. We must also consider the economic agenda, as well as issues of safety and efficacy in relation to CAM provision.

\section{A Gloomy View of Alternative Medicine}

\section{Knipschild, P.G.}

Maastricht University, The Netherlands

Suppose that I were the medical advisor of a new health care insurance company; what packet would I recommend? I think that I would make it rather small. Of course, I would start by abandoning all health care of dubious efficacy. Next, I would also leave out all health care of which the efficacy is not yet reasonably proven. For every diagnostic or therapeutic tool, one is obliged to first show me with sound research, including some rather large randomised blinded trials, that it is truly beneficial and does not have too many side-effects. Still, I would not stop there. For every disease with several efficacious treatments, my recommendation would be to at least start with the cheapest treatment, given by a qualified, but not too expensive practitioner.

The main problem of our regular health care is that too often it is rather costly. In many circumstances, simple diagnostic tools are good enough. The newer drugs are expensive and for many diseases not much better than the older ones. Moreover, all kinds of surgery could easily be taught, I think, to less qualified personnel than experienced surgeons.

With alternative medicine, the main problem is not its costs. Many alternative practices are not more expensive than their regular counterparts. Its main problem is its questionable efficacy. To me, tools such as medical astrology make no sense, whereas the invalidity of, e. g., iridology is documented in the literature. The same is true for the many fields of alternative therapeutics. Is there anyone who can show me nice trials that highlight the efficacy of paranormal healing? For every publication that seems to show the efficacy of acupuncture for a certain indication, I can give you another that is not so positive. Homeopathy remains odd, and rightly so, several recent trials have proven its inefficacy. Even the best herbal extracts (no extreme dilution) are not very useful, especially not if they are compared to the best 'chemical' drugs.

There may be a few undetected pearls, but generally, I think, alternative medicine does not work. Yet, I am fully aware of its popularity. If other people want to question my ideas or choose another health insurer, that is fine to me, as long as I do not have to pay for it.

\section{Methods to Enhance the Evidence - Randomized Clinical Trials}

Haskell, W.L.

Stanford University School of Medicine, Palo Alto, CA, USA

With the evolution of evidence-based medical practice, the randomized clinical trial (RCT) has taken center stage as the gold standard methodology for establishing a causal relationship between a therapy and clinical benefit. Many medical organizations developing clinical practice guidelines for both traditional and non-traditional therapies endorse only those therapies that have been shown to be effective when subjected to an appropriately designed and executed RCT. RCTs that are double blind with a placebo or usual-care control have demonstrated their effectiveness as a tool for evaluating specific pharmacological therapies and frequently have been considered the preferred methodology for determining the clinical benefit of life-style or other therapies. An RCT can help eliminate confounding by baseline variables (by randomization) and post-randomization confounding such as unintended interventions (by blinding) that are difficult to control with other study designs. Randomization can help eliminate a biased effect due to both identified and non-identified confounding variables. Variations in RCT methodology that should be considered for testing therapies include the run-in design, the factorial design, randomization of matched pairs, prerandomization and group randomization. However, the use of RCTs is limited when therapies can not be blinded, the placebo interacts with the active treatment, the therapist-patient interaction is critical to the therapy, a placebo or alternative treatment would be inappropriate, or when the clinical event of interest is rare. Of particular concern has been the many facets of the 'placebo effect' that can not be disaggregated from one another and can interfere with the correct interpretation of an RCT. A decision regarding the use of an RCT for evaluating a medical therapy should be based on ist appropriateness for testing a specific hypothesis and not on the source of the therapy. It is probably worthwhile to determine for each promising therapy if a properly executed RCT is warranted and appropriate for establishing the nature and magnitude of its effects (both risks and benefits) for a specific clinical outcome. If an RCT is not appropriate methodology, other study designs need to be instituted that, along with supportive data, can demonstrate causality of benefit at an acceptable risk.

\section{Problems of Randomised Treatment Studies in Complementary Medicine}

\section{Gerhard, I.}

Outpatient department of complementary and alternative medicine (CAM), Women's Clinic, University of Heidelberg, Germany

Background: Since the inauguration of the outpatient department for complementary medicine in 1993, randomised treatment studies were given highest priority in our research. With the experiences of the past years, however, it is questionable whether energy and expenditure invested into such studies bear a reasonable relation to the produced results. Example 1: In a prospective randomised study we examined the question of hormone-dependent infertility and sterility of unknown reasons and whether homeopathic treatment could be applied with positive results in these patients unsuccessfully treated by classical methods. During 20 months of recruitment, 1,800 infertile patients were seen, 300 of which corresponded to the eligibility criteria (16\%). Only 28 women accepted to be randomised $(1.5 \%$ of patients wishing children, $9 \%$ of all women eligible for the study). In regard to the main endpoint only 18 patients (64\% of the randomised study participants) could be evaluated after one year.

Example 2: In an ongoing prospective mistletoe feasibility study, breast cancer patients were treated by standard methods and complemented by mistletoe injection or placebo. During a six-month period, 424 breast cancer surgeries were performed. 48 or $11 \%$ of the intention-to-treat-women were willing to take part in the mistletoe study, half of whom fulfilled the eligibility criteria. However, only 8 women or $2 \%$ agreed to be randomised.

Conclusions: Highly informed patients, widespread application and predetermined choice of alternative and complementary methods of treatment, and individual decisions for certain treatment options, are the main obstacles that make studies, corresponding to established rules of biometrical and epidemiological standards, more and more dif- 
ficult in the field of alternative medicine. New methods of recruiting patients, of implementing and carrying out studies have to be developed to test treatments in the field of complementary and alternative medicine.

\section{What Are Non-Randomized Trials Good for?}

Weidenhammer, W.; Melchart, D.

Centre for Complementary Medicine Research, Dept. Internal Med. II, Technische Universität Munich, Germany

There is a broad variety of different methodological tools to get knowledge on scientific phenomena. The crucial criterion to decide which tool is the most appropriate is the kind of question you wish to answer. If the question is to prove the causality of specific effects, RCTs undoubtedly are the first choice. When evaluating health care or the quality of different providers of health services, for example, non-randomized trials might be the better choice. For each set of circumstances it is to analyze thoroughly whether randomization is

- possible,

- non-misleading,

- appropriate or,

- necessary.

On the one hand, there are a lot of ways to minimize the weaknesses of non-randomized trials like controlling for identified confounding variables or to adjust for baseline imbalances. On the other hand, this kind of trials bears advantages concerning an adequate balance of internal and external validity. In complementary medicine (often in the case of chronic diseases) the patient himself commonly prefers and demands a specific treatment. Trials which take into account this fact facilitate the generalizability of their findings to the population of interest.

Complementary Medicine does not need special research methods. The scientific tools are available yet. For a given question it is the challenge to find the most appropriate mixture out of them.

By means of own empirical studies the development from a simple observational study to a comprehensive clinical data base allowing comparisons of different treatments by different providers on predefined subgroups of patients will be demonstrated.

\section{The Nature of Adverse Events in Complementary Medicine: Chinese Medicine as a Case Example}

Bensoussan, $A$.

Research Unit for Complementary Medicine, University of Western Sydney, Campbelltown, Australia

Background and Objectives: With the growing usage of complementary medicine $(\mathrm{CM})$, regulatory authorities, health professionals and the public require a good understanding of the risks presented by its practice. Adverse events related to $\mathrm{CM}$ are varied and not infrequently reported. However, despite all the case reports and summaries of these reported events, little is understood about the relative risk to the public of the practices of CM compared to other health professions. In order to develop an understanding of the comparable risks it is necessary to establish data on the frequency of such events in clinical practice. Methods: Data on adverse events in traditional Chinese medicine (TCM) are presented as a case example. Comprehensive data were collected by survey of members of all occupational health groups, registered and unregistered, who practise TCM or one of its modalities. Adverse event reports were also analysed to determine the categories of risks that existed. Results: Adverse events occurring as a result of the practice of TCM are often reported by practitioners. These may be due to the actual administration of TCM therapy (such as injuries caused by acupuncture and toxic reactions to herbs), due to variable quality of medicinal products (such as poor manufacturing, substitution or adulteration of herbs), or a result of poor clinical judgement of practitioners (such as misdiagnosis). TCM practitioners experience one adverse event every eight to nine months of full-time practice. Conclusions: Comparison of adverse event rates in TCM with reports from other health professions indicates that TCM may be relatively safe compared to Western medicine, although it may pose greater risks than some currently regulated health care practices. This may also be true for other complementary medicine fields. Consideration needs to be given to distinctions between adverse events and anticipated healing responses. An analysis of the risk to benefit of the therapy is also important.

\section{The Safety of Complementary Medicine: An Overview of the Available Evidence}

Ernst, E.

Department of Complementary Medicine, School of Postgraduate Medicine and Health Sciences, University of Exeter, UK

One of the many and complex reasons for complementary medicine's (CM) current popularity is the notion that it is inherently safe. This is naïve at best and dangerously misleading at worst. The indisputable facts are as follows:

- no therapeutic intervention can ever be entirely risk-free,

- very little research on the safety aspects of $\mathrm{CM}$ has been carried out,

- thus much uncertainty exists in this area.

This paper will deal with various safety aspects of some of the most prevalent forms of CM. It is helpful to differentiate direct risks from indirect risks. Direct risks pertain to the safety of the therapy while indirect risks relate to the safety of the therapist. Examples of both will be provided. There are several important obstacles to reliable risk assessments of CM:

- the reporting systems known from conventional medicines do not cover many areas of CM,

- thus we often rely on inconclusive evidence, e. g. from anecdotal reports which might only form the 'tip of the iceberg',

- the CM community is often emotionally opposed to discussing safety issues of $\mathrm{CM}$,

- it is extremely difficult to find funds to research this area.

In the final analysis it is not relevant to look at safety in isolation, or to compare the (low) risks of $\mathrm{CM}$ with the (high) risks of conventional medicine. The questions that ultimately require answering are: does a given therapeutic intervention do more good than harm? (e.g. a risk/ benefit analysis), and is one (complementary) treatment associated with a more favourable risk/benefit profile than another (conventional) therapy? (e.g. a relative risk/benefit analysis). At present the evidence is far too incomplete to even attempt conclusive answers to these questions. It follows that more research into the safety aspects of CM is badly needed - after all, 'primum nihil nocere' is an essential overriding principle of any type of medicine. 
Effects of Homeopathy and Acupuncture in General Practice Intermediate Results of a Longitudinal Observational Study as Seen in Work-Absenteeism

\section{Walach, H.; Güthlin, C.}

University Hospital, Dept. of Environmental Medicine and Hospital Epidemiology, Freiburg, Germany

Background: We set out to document use of and long-term effects of homeopathy and acupuncture, when offered widely by an insurance company. Methods: Prospective documentation of 5,000 patients insured with a public insurance company (Innungskrankenkasse) who are provided with free treatment of acupuncture and homeopathy. All patients are followed up from the beginning of treatment for 4 years, according intent to treat. Doctors provide data on each visit, patients fill in questionnaires including MOS SF-36 at the beginning and at the end of treatment, as well as each year after the end of the treatment. Insurance data on work-days-lost are provided by the insurance company. Results: By now, 1,000 patients have been entered into the analysis, 400 patients had work-days lost between 1 day and 1.5 years and more than 4,000 cases of absenteeism over the period of 1991 to 1999. There is an increase of work-days lost until 1997 followed by a decrease, a result reflecting the general trend in Germany. Patients seeking homeopathy or acupuncture had more work-days lost at the beginning of the treatment than the rest of the insured population. They experienced a significant reduction over the course of the observation and had an amount of work-days lost at the end of the observation period comparable to that of the general insured population. Health-related quality of life (SF 36) increased significantly by about 10 points and decreased slightly again to follow-up due to conservative extrapolation of missing data, but staying above initial values. Conclusions: We conclude from our preliminary results that patients using homeopathy and acupuncture show significant improvements in their health-related quality of life, which reflects in a significant reduction of work-days lost. It remains to be seen whether these data will be corroborated by our complete sample at the end of the full observation period.

\section{'Quality Management and Research' as a Pragmatic Program to Evaluate and Improve Complementary Medicine in Practice - Rationale, Concept, Results}

\section{Melchart; D.; Weidenhammer, $W$.}

Centre for Complementary Medicine Research, Dept. of Internal Medicine II, Technische Universität Munich, Germany

In Germany there exists a considerable number of in-patient facilities specialized in providing various complementary therapies in addition to conventional basic care. In these hospitals mainly chronically ill patients are treated with a whole range of complementary methods, depending on the concept, preferences and availability of the respective hospital. For many of the methods used there is little or no evidence from randomized controlled trials; the combinations have rarely been investigated at all. It seems illusory to expect that the effectiveness of a representative sample of such 'therapeutic cocktails' will be evaluated in randomized studies. The investigation of single components allows limited conclusions on complex strategies. In this situation - lack of scientific evidence on one side and strong interest of patients on the other - pragmatic approaches have to be found to provide at least reliable basic information about what is going on in a defined hospital. For this purpose the concept of 'quality management and research' has been developed. The program links elements of quality assurance and 'conventional' scientific research. Quality assurance is reflecting the fact that the interventions are already widely used and have to be further optimized. It is provider-oriented, creates transparency and gives confidence that an intervention is delivered by a certain provider in a qualified manner. Research is reflecting the lack of evidence, is looking for generalizable answers, and will show whether the intervention works.

In a step-by-step strategy the concept gives information on quality aspects on several levels for and about patients and providers. Therefore, special 'quality-profiles' of the hospital infrastructure, the patients, processes and outcomes are determined in the respective setting. The provider-oriented 'profile' gives a pragmatic basis for decision-making to patients ('Do I want to be treated in this certain hospital?'), health care professionals ('Should I refer my patient to the hospital?') as well as insurers ('Should we reimburse the costs for a stay in this hospital?') and politicians ('Does the hospital meet a minimun standard and should it be part of the providing system?'). Projects to improve the quality have to be identified, organized and evaluated. In a next evaluative step the attainment of preinterventionally defined goals has to be investigated. Finally, the profiles of different providers (hospitals) are compared. The last step of the program is to enhance the evidence for specific interventions, to test hypotheses and to perform literature reviews. The steps will be illustrated by own results. In addition, an electronic patient documentation is piloted which will allow assessments in routine conditions, transfer of information between providers, and evaluation.

\section{Monitoring Outcomes in Routine Clinical Practice}

Long, $A$.

University of Salford, UK

Background: The pursuit of evidence-based practice requires the adoption of findings from high-quality research within real life choices about who, when and how to treat and provide care for individual service users. Despite the wide and continuing debate over the relevance of particular research methods to evaluate complementary and alternative medicine (CAM), less attention has been given to what success/outcome criteria and measures to use, whether these have a sufficient breadth in perspective and time scales for measurement. Furthermore, given the noted distinction between efficacy and effectiveness, the CAM (and other) practitioner must monitor the emerging outcomes (are they as good as the research evidence would suggest they should be?) with a view to develop appropriate action to enhance the achievement of best outcomes. Methods: The work is founded on an established and systematic framework for exploring and monitoring outcomes within routine practice [1]. Results: Central to outcomes measurement within routine settings is the need to address the desired outcomes of the therapy user and practitioners. While in CAM considerable attention has been given to measuring the subjective experience of therapy users, left unmeasured is the notion of holism, in particular, the measurement of the effect of the interaction between therapist and therapy user and its underlying philosophy of health and healing. The measured effects of CAM treatments may thus be credited with a lesser effect than is achieved in reality. To undertake outcomes measurement within real life settings, measures must not only be psychometrically sound (reliable, valid and, for outcomes measurement, responsive to change) but also be feasible to use within routine practice. The latter suggests the need for the use of short(er) measures and that mirror and/or collect information seen as clinically useful to monitor the ongoing health and healing of the service user. Conclusion: CAM practitioners have a duty to base their practice on up-to-date and appropriate research evidence and to ensure that high-quality care is provided to service users. Appropriate measures must be used and emerging outcomes monitored within real life settings.

\section{Reference:}

Long AF: Managing for outcomes. J Integrated Care 1999,3:162-170. 


\section{Are Statistical Significance and Effect Sizes Enough? Methods to Describe Clinical Relevance of Continuous Outcome Data for Single Patients}

Schuck, $P$.

FBK Research Institute, Bad Elster, Germany

Conclusions of efficacy/effectiveness studies as well as quality management studies are usually drawn from inferential statistics (e. g. Student's $\mathrm{t}$-test). Nowadays effect sizes are additionally demanded, because inferential statistics may be very misleading, especially in the case of large samples.

However, both methods focus on groups of patients. In psychotherapy research Jacobson et al. [1] developed techniques to assess 'clinical significance' of outcome data for single patients, when instruments like the SF-36 are used:

Subjects are only rated as 'recovered' if their single difference score is statistically different from measurement error (the so-called 'Reliability Change Index') and if their post-treatment score has returned to values of 'normal functioning'. Three different cutoff points are proposed for the latter (assuming that higher values indicate better functionality): (a) The post-treatment score falls outside the range of the dysfunctional population. Range is defined as extending to 2 standard deviations (SD) above (i.e., in direction of more functionality).

(b) The post-score falls within the range of functionality of the normative sample, where range is defined as beginning at 2 SDs below the mean of the normal population.

(c) The post-score is higher than the 'mean' between the two distributions.

Whereas (a) can also be computed if there are no normative data, the choice between (b) and (c) depends e. g. on the amount of overlapping between the two distributions.

These techniques are demonstrated and modifications are discussed if certain assumptions are not met for data within complementary medicine.

\section{References:}

Jacobson NS, Roberts LJ, Berns SB, McGlinchey JB. Methods for defining and determining the clinical significance of treatment effects: description, application, and alternatives. J Clin Consult Psychol 1999;67:300-307.

\section{Addition (Late Submission) for the Following Section with Peer-Reviewed Abstracts}

\section{Effect of Acupuncture for Smoking Cessation}

Dong, H.; Berg, J.E.; Høstmark, A.T.

Department of Preventive Medicine, University of Oslo, Norway

Background: This study was undertaken to examine the effects of acupuncture for smoking cessation and to examine whether some acupoints are more effective than other for smoking cessation. Methods: Altogether 46 healthy men and women, $39 \pm 9$ years of age, who smoked $20 \pm 6$ cigarettes per day and who had smoked for $23 \pm 8$ years. The subjects were randomly assigned to a test group (TG) or to a control group (CG) depending on whether the acupoints used were assumed to be anti-smoking acu-points (TG) or to have no effect for smoking cessation (CG). The acupuncture treatment was a combination of body electroacupuncture, ear acupuncture and ear acupressure. Before each treatment and after the last treatment subjects answered questionnaires about their smoking habits and attitudes. The concentrations of serum continine, serum thiocyanate, serum peroxides and plasma fibrinogen were measured before the first and after the last treatment and 8 months later. Results: The daily cigarette consumption fell in both groups, but the reduction was larger for TG than for CG $(\mathrm{P}<0.002)$. Altogether $31 \%$ and $23 \%$ of subjects in TG had quit smoking completely at the end of the treatment and 8 months later, compared with none in CG. For TG the concentrations of continine and thiocyanate were reduced significantly after the treatment period $(\mathrm{P}<0.001)$, but no reductions were observed for $\mathrm{CG}$. For both groups the taste of tobacco worsened during the treatment period, but the effect was more pronounced for TG than CG $(\mathrm{P}<0.05)$. The desire of smoking fell significantly in both groups after treatment, and the effect was larger for TG than for CG $(\mathrm{P}<0.001)$. No significant changes in serum peroxides and plasma fibrinogen concentrations were observed. Conclusions: This study suggests that acupuncture may help motivated smokers to reduce their smoking or even quit smoking completely. Different acupoints appear to have different effects for the smoking cessation. 


\section{Abstracts Peer-Reviewed}

\section{A Meta-Analysis of Effects of Garlic on Serum Lipids}

\section{Ackermann, R.T.; Mulrow, C.D.; Ramirez, G.}

University of Texas Health Sciences Center, San Antonio, Texas, USA

Objective: To assess whether oral garlic supplementation improves serum lipid profiles in adult humans. Methods: We searched 1800 English and non-English citations through December 1999 using 11 electronic databases (e.g., MEDLINE, EMBASE, the Cochrane Library, NAPRALERT), article bibliographies, manufacturers, and experts. Search terms included: aglio, allium sativum, garlic, garlic extract, garlic oil, knoblauch, kwai, kyolic, and 17 additional related chemical terms. Selection criteria were randomized trials of at least 4 weeks duration that measured lipids and compared garlic with placebo, no garlic, or another active agent. Two independent physicians abstracted data from trials. Lipid outcomes were pooled using standardized mean differences. Results: Of 41 trials meeting selection criteria, 1 had clearly adequate randomization processes; 31 used double-blind designs; and 4 had $\geq 20 \%$ drop-outs. Trial interventions included standardized dehydrated garlic preparations such as Kwai or PurGar $(n=22)$, aged-garlic extract $(n=3)$, garlic oils $(n=6)$, raw garlic $(n=1)$, various non-standardized dried preparations $(\mathrm{n}=7)$, and combination products $(\mathrm{n}=2)$. Pooled analyses of placebo controlled trials showed that garlic preparations significantly reduced total cholesterol by $9.9 \mathrm{mg} / \mathrm{dl}$ (CI 1.7 to 18.2$)$ at one month and by $20.4 \mathrm{mg} / \mathrm{dl}(95 \%$ CI 12.4 to 28.5$)$ at 3 months. Combining only those studies that evaluated standardized dried preparations showed total cholesterol reductions of $14.1 \mathrm{mg} / \mathrm{dl}$ (CI 1.7 to 18.2 ) at 1 month and $27.4 \mathrm{mg} / \mathrm{dl}$ (CI 18.2 to 36.5 ) at 3 months. Six trials had six-month outcomes; pooled analyses showed no significant reductions in total cholesterol. Patterns of pooled analyses of triglyceride and low density lipoprotein cholesterol paralleled total cholesterol analyses, but were not statistically significant. No significant changes in high density lipoprotein cholesterol levels were found. Conclusions: Randomized trials consistently show that garlic leads to modest short-term reductions in total cholesterol. Data regarding long-term effectiveness, superiority of particular preparations, and relevance to actual clinical outcomes are inconclusive. Unclear randomization procedures and inadequate blinding limit applicability of these findings. Additional trials of longer duration that better control for these methodological shortcomings are warranted.

\section{Traditional Chinese Medicine (TCM) in Diagnosing Frequent Recurring Cystitis in Adult Women}

\section{Alrek, T.; Aune, A.; Barheim, A.}

Division for General Practice, Department of Public Health and Primary Health Care, University of Bergen, Norway

Background: Frequently recurring acute cystitis in adult women is a common entity in Western medicine. A recent study has shown that acupuncture seems effective in its prophylaxis. In prophylactic treatment the women are treated in between attacks of acute cystitis to prevent recurrences. Objectives: In this study we wanted to describe the frequency of different TCM diagnoses used on this situation, and to evaluate in light of the theoretical TCM foundations which symptoms and signs most often form the base for TCM diagnoses used on this en- tity. Methods: A descriptive study based on TCM diagnostic methods as used by experienced acupuncturists, and a questionnaire on symptoms used in TCM diagnostics. Results: Of the 61 women with frequently recurring cystitis, $90 \%$ were diagnosed as having either a Spleen/Kidney yang xu/qi xu, or a Liver qi stagnation. Only $10 \%$ fell in other diagnostic groups. Of all symptoms noted, only three differed in frequency between the two main diagnostic groups: feeling cold (29/33 v. $13 / 22, p<0.05)$, feeling tired $(25 / 33$ v. $4 / 22, p<0.001)$, and having a preference for sweets $(10 / 33$ v. $2 / 22, p<0.05)$. Conclusion: Our findings indicate that recurrent cystitis may be a useful case for further research on TCM. They also have implications for TCM-based diagnostic work on this entity.

\section{Qigong for School Children - A Controlled Pilot Study}

Becker, M. ${ }^{1}$; Witt, C. ${ }^{1}$; Bandelin, K.; Schulze, D. ; Binting, S. ; Willich, S.N. ${ }^{1}$

${ }^{1}$ Institute of Social Medicine and Epidemiology, Charité Hospital, Humboldt University of Berlin, Germany

Background and Objectives: The effects of qigong exercises have not been determined systematically. The present study aims to evaluate the effects of qigong lessons on the achievements at school, social behaviour and health in school children. Methods: In a controlled intervention study, all pupils of 4 classes at two schools (one primary school and one secondary school) were included into the evaluation. One class of each school obtained qigong lesson for 6 months. For each class, a control class in the same age group participated in the evaluation without qigong lessons. Standardised questionnaires were used by teachers, parents and pupils to obtain information on concentration, creativity and social behaviour at the beginning of the study and after 6 months. To assess quality of life we used the KINDL questionnaire. Results: A total of 90 pupils have been documented ( $52 \%$ boys, $48 \%$ girls). 40 pupils were at the primary school (20 in each class, age $7.4 \pm 0.5)$ and 50 at the high school ( 25 in each class, age $13 \pm 0.8)$. At both schools, the pupils practised qigong exercises at least three times a week for 20 minutes. The score of the teachers questionnaire improves for the qigong group $(55 \pm 9$ to $59 \pm 9, \mathrm{p}<0,001)$ whereas no change was shown for the control group ( $56 \pm 7$ and $56 \pm 8$, ns). Furthermore, no difference was observed for both groups by the teachers questionnaire and the KINDL. Conclusion: To our knowledge, the present study is the first to evaluate the effectiveness of qigong lessons in school children. Within 6 months after initiation of qigong lessons, concentration, creativity and social behaviour improved for the qigong group. However the differences we detected are small, further long-term follow-up studies with larger sample sizes are needed.

\section{The Mechanism of Action of Watery Peat Extract on the Spontaneous Contractile Activity of Smooth Muscles}

Beer, A.M. ${ }^{1}$; Lukanov, J. ${ }^{2}$; Sagorchev, $P^{2}$

${ }^{1}$ Klinik Blankenstein, Hattingen; ${ }^{2}$ Institute for Medical Hydrology, Bad Kissingen, Germany

Background and Objectives: In the literature there are reports about the effects of peat and its products on the spontaneous contractile activity (SCA) of smooth muscles. In our recent work we have shown that watery peat extract is a stimulus to the SCA of smooth muscle fibres. These effects, caused by watery peat extract, changed to self-blocking effects after longstanding (4-5 hours) influence. The mechanism of action is not obvious at this moment. The purpose of this study is to elucidate the peat mechanism of action. Methods: The effects of watery peat 
extract of SCA from smooth muscle under normal conditions and in presence of different receptor blocking substances have been studied. The SCA were registrated by tension transducers under isometric conditions. Results: Our results show that blocking at the same time $\alpha_{2}$ adreno- and $\mathrm{D}_{2}$ receptors the stimulating activity of watery peat extract of SCA of smooth muscle was suppressed. The self-blocking actions on the stimulating activity of watery peat extract of smooth muscle SCA were interconnected by blocking $\alpha_{2}$-adreno- and $\mathrm{D}_{2}$ receptors. Conclusion: The watery peat extract possesses biologically active substances which show partial agonist effects of $\alpha_{2}$-adreno- and $\mathrm{D}_{2}$ receptors.

\section{Analgetic Effect of Acupuncture in Endometriosis}

\author{
Blank, A.; Li, P.; Freivogel, K.P.; Schön, J.; Gerhard, I.
}

Ambulanz für Naturheilkunde, Gynäkologische Endokrinologie und Fertilitätsstörungen der Universitäts-Frauenklinik Heidelberg, Germany

Background: Endometriosis is a complex disease influenced by genetic, immune, hormonal and environmental factors. The most frequent symptoms in endometriosis are infertility and pain. Prevailing treatment is surgical and hormonal. Objective: Reduction of pain by acupuncture. Method: Retrospective observational study to evaluate effectiveness of analgetic treatment by acupuncture. 33 postsurgical and posthormonal patients suffering from endometriosis-typical pain filled a self-administered questionnaire with a categorial pain scale (1-5). Weekly treatment by acupuncture was performed over three months. Results: 25 patients could be evaluated, 8 were lost to follow-up. 20 patients experienced substantial relief of pain and improvement of general well- being during acupuncture treatment. 13 patients could be followed up until 3 months after cessation of acupuncture therapy. All still reported reduction of pain and improvement of general well-being in comparison to the pretreatment period. Conclusion: Acupuncture is a suitable method to treat the complex pain-symptomatology of endometriosis. A study with a larger number of patients suffering from endometriosis is warranted.

\section{Setting the Research Agenda: Qualitative Research of Patient Data in Homeopathy}

Brands, $M^{1}{ }^{1}$; Weatherley Jones, $E^{2}$

${ }^{1}$ Free University Amsterdam, NL; ${ }^{2}$ University of Sheffield, UK

Background: Homeopathic materia medica has been established before modern cognitive frameworks and epidemiological techniques were developed. This has led to a materia medica which is not scientifically structured with regard to 1) The origin of the composing symptoms and 2) The criteria of newly added symptoms and features of patients. This reduces the accuracy of the reference of a single symptom to remedy pictures, the disease category in homeopathy. An improvement in this situation may dramatically increase the accuracy and speed of prescription in homeopathic practice. Methods: The model uses instruments from decision making strategy, linguistics and epidemiology [Checkile \& Cooke 1999, Lakoff \& Johnson 1980, Rosch \& Mervis 1975]: 1) semantic analysis: a) texts from materia medica literature are computersearched for metaphors linking clusters of symptoms; synonyms and thesaurus programmes are used; b) salience and frequency of certain words are assessed to identify central metaphors. 2) experimental analysis: a) which data in the materia medica derive from toxicology, provings (experiments), and clinical successes, respectively? b) Can subclusters within these data be identified to stem from individual patients? c) Which criteria of clinical success were used for inclusion of symp- toms in the remedy picture? Results: 1) Identification of central metaphors can, if compared with the actual patient's narrative, make diagnostic strategies in homeopathic consultations explicit, e. g. 'triggers' to think of certain remedies 2) Calculation of sensitivity, specificity and predictive value of symptoms within some frequently used homeopathic remedies (retrospective analysis). The model then serves for prospective studies. 3) Metaphors are linked to the quantitative data by 'decision in uncertainty' models. 4) After the establishment of this model for a few remedies, an international network will be formed to extend the study to many remedies. Conclusion: Qualitative techniques can be used to quantify data in the homeopathic materia medica in order to make this database accessible for scientific research from other disciplines and to improve clincal practice.

TCM Therapy with Acupuncture and Herbal Medicine in the Treatment of Patients with Allergic Rhinitis: A Randomized Controlled Trial

TCM Study Group*: Seufert, J. ${ }^{1,2}$; Schuppan, D. ${ }^{1}$; Nögel, $R^{2}$; Leohnardi, H. ${ }^{2}$; Kohnen, R. $^{3}$; Joos, $S^{1}{ }^{1}$; Hummelsberger, $J^{2}$; Hempen, ${ }^{2}{ }^{2}$; Hahn, E. G. ${ }^{1}$; Brinkhaus, B. ${ }^{1}$ (in alphabetical order) $*^{1}$ Dept. of Complementary Medicine, Dept. of Medicine I, University Erlangen-Nürnberg; $*^{2}$ Internationale Gesellschaft für Chinesische Medizin, München; ${ }^{*}$ Institute of Medical Research Management and Biometrics, Nürnberg, Germany

Background: There has been a marked increase in the prevalence of allergic rhinitis in countries with a Western life-style. To date, no effective treatment has been found, and complementary medicine is becoming ever more popular. We investigated the efficacy and side effects of adjuvant acupuncture (A) or Chinese herbal medicine (CHM) administered in accordance with the rules of traditional Chinese medicine (TCM) in patients with allergic rhinitis. Methods: In this randomized, single-blind controlled study, 52 patients with allergic rhinitis received either A and CHM administered in accordance with the rules of TCM (TCM group), or A applied to non-acupuncture points together with an unspecific CHM (control group). The patients were treated for a total of 6 weeks - A was applied $1 \times$ weekly, CHM was taken $3 \times$ daily. The target parameters were a symptom questionnaire (SFB, Walker), the Rhinitis Life Qualitiy Questionare (RQLQ, Juniper), rhinomanometry (RMM), visual analog scale (VAS) and the Clinical Global Impression Scale (CGIS). Results: The TCM group and the control group showed no significant differences in term of age, gender and duration of illness. After 6 weeks of treatment, the TCM group showed a significant improvement in the VAS, CGIS and the RQLQ, but not in the SFB and the RMM, in comparsion with the control group. There were no differences in side effects among the treatment groups. Conclusion: TCM is effictive in improving a number of subject parameters in patients with allergic rhinitis. Side effects were not seen in any of the groups. Additional studies involving lager numbers of patients with allergic rhinitis are needed to confirm the therapeutic effect of TCM treatment observed in this study. 


\section{Analgesic Effects of Carbon Dioxide Insufflations as Adjunct Therapy in Persons with Non-Specific Neck or Low Back Pain - An Open,} Randomised, Controlled, Clinical Tria

Brockow, ${ }^{1}{ }^{1}$; Dillner, A. ${ }^{2}$; Franke, A. ${ }^{1}$; Resch, K.L. ${ }^{1}$

${ }^{1}$ Saxon Balneology and Rehabilitation Sciences Research Institute, Bad Elster

${ }^{2}$ Rehabilitation Hospital Sachsenhof, Bad Elster, Germany

Background and Objectives: In Central Europe subcutaneous carbon dioxide insufflations (SCIs) are used as serial treatment modality for pain control in non-specific neck and low back pain. Up to now only uncontrolled observational trials exist for these indications. The following randomised controlled trial aimed to evaluate the analgesic effects of SCIs in non-specific neck or low back pain. Methods: 140 consecutive patients of a rehabilitation hospital with non-specific neck and/or low back pain (mean age 65 years) were allocated by external telephone randomisation to SCIs (10 treatments in 12 days; maximal dose: $200 \mathrm{ml} \mathrm{CO}$ s. c.) or to no SCIs. All patients received a complex, standardized in-patient rehabilitation programme. Main outcomes were affective and sensory pain perception ('validated German Language questionnair' - SES) as well as pain intensity (VAS). Outcomes were assessed before, after 5 and after 10 treatments. Statistical analysis was done by means of repeated-measures ANOVA using intention-to-treat strategy and incorporating predictors identified by multiple regression models. Controls received the study intervention after completion of the trial. SCIs were administered by 2 physicians in randomised order. Results: Demographics, descriptive clinical variables, and outcomes were well balanced. Independent of group allocation and locus of pain, highly significant $(\mathrm{p}=0.000)$ longitudinal changes were found for all outcomes. SCIs, however, did not produce additional beneficial effects compared to standard treatment (group differences: $\Delta$ affective pain perception: -2.2 [ $95 \%$ CI: -5.2 to 0.9 ]; $\Delta$ sensory pain perception: -1.2 [95\% CI: -3.0 to 0.7 ]; $\Delta$ pain intensity: -6.5 [95\% CI: -14.0 to 1.0$]$ ). Within-group effect sizes were moderate to large (0.5 to 1.1$)$, whereas between-groups effect size differences varied only between 0.2 and 0.3 . Conclusion: No additional, clinically relevant effects of SCIs could be found in a sample of older people with non-specific neck and/or low back pain. Further investigations should deal with SCIs in a monotherapeutical setting and within a sample of a younger population.

\section{The Depth of Needle Insertion as a Variable of Stimulation Intensity. Two Randomized Controlled and Blind Clinical Studies.}

Ceccherelli, F.; Gagliardi, G.; Rigoni, M.T.; Ruzzante, L.; Giron, G.P. Observatory on Complementary Medicine, Anesthesiological Section of the Department of Pharmacology and Anesthesia, University of Padova, Italy

Objective: To compare the effect of superficial vs. deep acupuncture in patients affected by lumbar and shoulder myofascial pain. Methods: Two randomized controlled and blinded trials were performed. The low back pain group was formed by 42 patients divided into 2 groups of 21 each. In one group superficial acupuncture was performed with a 2-mm needles insertion; in the other group acupuncture was performed at the muscular level. 8 sessions were administered of 20 minutes each with manual stimulation of the needles for 1 minute at the beginning of the session and for 20 seconds every 5 minutes. All patients were subjected to the same bilateral therapeutic pattern: Shiqizhui (Extra 19), Jizhong (VG 6), Yangligquan (GB 34), Zhibian (UB 54), Shenmai (UB 62) and the needle insertion in 4 trigger points (TP) or in 4 most painful muscular tender points (as second choice) found in the lumbar area. The shoulder pain group included 44 patients of both sexes di- vided into two (superficial and deep) groups of 22 each. All patients were treated with 2 acupunctural sessions weekly for 5 weeks (10 sessions) of 20 minutes each utilizing the following bilateral acupoints: (Houxi) 3 SI, (Quchi) 11 LI, (Jianyu) 15 LI, (Jianliao) 14 TW, (Jianzhen) 9 SI, (Tianliao) 15 TW, (Dazhui) 14 VG. Pain was measured with the Mc Gill Pain Questionnaire before therapy, at the end of it and after three months. Results: The total pain scores in low back pain patients were $22.2 \pm 16.1$ in the superificial acupuncture group and $14.5 \pm 10.9$ in the deep acupuncture group at the end of the therapy. After three months the values were $18.0 \pm 17.2$ and $7.5 \pm 12.9$, respectively. The total pain scores in shoulder pain patients were $12.86 \pm 11.4$ in the superificial acupuncture group and $4.91 \pm 8.2$ in the deep acupuncture group at the end of the therapy. After three months the values were $18.2 \pm 7.2$ and $7.2 \pm 5.8$, respectively. Conclusions: Deep acupuncture seems to be more effective in the treatment of lumbar and shoulder myofascial pain in comparison to superficial acupuncture. Publications of clinical trials of acupuncture should report all the details concerning needles stimulation to warrant the reproducibility of the investigations on this topic

\section{Acupuncture Massage vs Swedish Massage and Individual Exercises vs Group Exercises in Low Back Pain Sufferers - A Randomized Clinical Trial in a $2 \times 2$ Factorial Design}

Franke, A. $^{1}$; Gebauer, S. $^{2}$; Franke, K. $^{2}$; Brockow, $T^{1}$

${ }^{1}$ FBK Research Institute, Bad Elster; ${ }^{2}$ In-patient rehabilitation hospital (Park Reha-Klinikum) Bad Gandersheim, Germany

Objective: Rehabilitation programs for low back pain (LBP) sufferers almost always contain physical exercises and various types of massage treatment. This study aimed at quantifying the efficacy of individual exercises (IE) versus group exercises (GE) and therapeutic 'Acupuncture' massage (APM; i. e. tonic stimulation of whole meridians) according to Penzel versus Swedish massage (SM) in LBP sufferers. Methods: One hundred and ninety patients participating in a complex in-patient rehabilitation program including physical exercises and massage treatment were randomized to four groups in a $2 \times 2$-factorial design. Main outcome measures were activities of daily living (Functional Questionnaire Hanover $-\mathrm{FFbH})$ and pain intensity $(10 \mathrm{~cm}$ visual analogue scale - VAS). Pre/post changes were evaluated by means of 2-way analysis of variance (ANOVA). Lumbar motility measured by a 2-inclinometer technique served as descriptive outcome. Results: Because of some differences between groups at baseline, standardized outcomes were used for analysis. APM showed beneficial effects for both disability and pain compared with SM (group differences: $\triangle \mathrm{FFbH}: 7.0 \%$ [95\% CI: 2.5 to 11.6$], \mathrm{p}=0.003$; $\Delta$ VAS: $8 \%$ [95\% CI: 2 to 15 ], $\mathrm{p}=0.024)$. Effect sizes of $\mathrm{e}_{\mathrm{FFbH}}=0.5$ and $\mathrm{e}_{\mathrm{VAS}}=0.8$ were found for $\mathrm{APM}$, as opposed to $\mathrm{e}_{\mathrm{FFbH}}=0.1$ and $\mathrm{e}_{\mathrm{VAS}}=0.4$ for SM. No significant group differences were found between exercise groups $(\Delta \mathrm{FFbH}: 0.5 \%$ [95\% CI, -4.2 to 5.2 ]; $\Delta$ VAS: $0.4 \mathrm{~cm}$ [ $95 \% \mathrm{CI},-0.3$ to 1.1$]$. Conclusion: Concerning the fact that commonly at best moderate effects are reported for 4-week in-patient rehabilitation programs in back pain sufferers the observed effect sizes with APM are promising and warrant further investigation in replication studies. In contrast to common view, no superiority of individual versus group exercises could be found in the present study. 
Long-Term Efficacy of Radon Spa Therapy in Rheumatoid Arthritis - A Randomized, Sham-Controlled Study and Follow-Up

\section{Franke, A. ${ }^{1}$; Reiner, L. ${ }^{2}$; Pratzel, H.G. ${ }^{3}$; Franke, T. ${ }^{1}$; Resch, K.L. ${ }^{1}$} ${ }^{1}$ FBK Research Institute, Bad Elster; ${ }^{2}$ In-patient rehabilitation hospital (Dr. Ebel Fachklinik) Bad Brambach; ${ }^{3}$ Institute of Medical Balneology and Climatology, Ludwig Maximilians University, Munich, Germany

Objective: Traditionally, spa therapy is used as an integral part of physical therapy for rheumatoid arthritis (RA). This study aimed at quantifying the efficacy of a series of baths containing natural radon and $\mathrm{CO}_{2}$ $\left(1.3 \mathrm{kBq} / 1,1.6 \mathrm{~g} \mathrm{CO}_{2} / 1\right.$ on average) versus artificial $\mathrm{CO}_{2}$ baths alone in patients with RA. Methods: Sixty patients participating in an in-patient rehabilitation program including a series of 15 baths were randomly assigned to 2 groups. Pain intensity (100 $\mathrm{mm}$ visual analogue scale) and functional restrictions (Keitel functional test, Arthritis Impact Measurement Scales - AIMS questionnaire) were measured at baseline, after completion of treatment, and 3 and 6 months thereafter. Aggregating the repeated measures of every patient, the mean change over time was used to evaluate the overall treatment effects. For confirmatory analysis Student's t-test for independent samples was applied. Results: Both groups showed a comparable baseline situation. After completion of treatment, relevant clinical improvements were observed in both groups without notable group differences. However, the followups revealed sustained effects in the radon arm and a return to baseline levels in the sham arm. After 6 months, marked between-group differences for both endpoints were found $(\Delta \mathrm{PI}=-16.9$ [95\% CI: -27.7 to -6.12 ], $\Delta$ AIMS $=0.57$ [95\% CI: 0.16 to 0.98$]$ ]). The between-group differences for both aggregated outcomes - pain and functional limitations - were statistically significant $\left(\mathrm{p}_{\mathrm{PI}}=0.04\right.$ and $\left.\mathrm{p}_{\mathrm{AIMS}}=0.01\right)$. Conclusion: Marked short-term improvements in both groups at the end of treatment may have masked potential specific therapeutic effects of radon baths. However, after 6 months follow-up effects were lasting only in patients of the radon arm. This suggests that this component of the rehabilitative intervention can induce beneficial long-term effects.

\section{Does Hydrotherapy Prevent Common Cold? Randomized Controlled Trial in Preschool Children with Frequent Common Colds}

Grüber, $C^{1}$; Riesberg, $A .{ }^{2}$; Mansmann, $U_{.}^{2}$; Wahn, $U^{1}$; Bühring, $M .{ }^{2}$ ${ }^{1}$ Charité Children's Hospital, Humboldt University; ${ }^{2}$ Benjamin Franklin University Hospital, Berlin, Germany

Background and Objectives: Few therapies have proved to be effective in prevention and treatment of common colds. Anecdotal reports suggest a possible effect of 'hardening' by hydrotherapy (systematic stimulation of the body surface with warm and cold water). This study was designed to compare hydrotherapy in addition to inhalation therapy with inhalation therapy alone for its effect on incidence and duration of common cold episodes in children. Methods: Both regimens were compared prospectively in a one-year clinical trial with randomized assignment of patients. 175 children, aged 3-7 years, with $\geq 6$ common cold episodes during the preceeding 12 months were assigned to either daily inhalation of normal saline (A, control group) or daily inhalation plus daily hydrotherapy (B, experimental group). Parents kept a daily diary about respiratory symptoms. Results: Groups did not differ at baseline with regard to age, sex, or cold episodes in the year before. Diaries were available from (A) 81 and (B) 65 patients. In the treatment period, there was no significant difference in the incidence of colds ( $A$ vs $B$, mean $\pm S D$, $4.8 \pm 3.5$ vs $4.1 \pm 3.3$ episodes), average duration of episodes ( $6.5 \pm 3.1$ vs $6.5 \pm 3.2$ days), cold episode days with earache ( $8.0 \mathrm{vs} 8.2 \%$ ) or sore throat $(20.7$ vs $20.2 \%)$, statistical significant differences were found for cough ( 93.3 vs $89.5 \%, P<0.001)$, rhinitis ( 95.6 vs $94.0 \%, P<0.05)$, and fever ( 9.9 vs $12.3 \%, P<0.01)$. Conclusion: This study demonstrates similar beneficial effects of normal saline inhalation and hydrotherapy in addition to inhalation among preschool children with frequent common colds. These findings do not provide support for the concept of 'hardening' as a preventive measure for colds.

Grant \# 01KT94/05, Federal Ministry of Education and Research, Bonn, Germany.

\section{Alternative Treatment of Patients with Advanced Cancer of the Pancreas}

Hager, E.D.; Dziambor, H.; Popa, G.; Höhmann, D.

BioMed-Klinik, Bad Bergzabern, Germany

Background and Objectives: Conventional therapies in patients. with advanced metastatic or recurrent adenocarcinoma of the pancreas have little impact on patient survival or quality of life. Objective, reproducible responses to single-agent or combination chemotherapy are $<10 \%$ and the percentage of symptomatic relief by treatment with 5-FU or gemcitabine is $5 \%$ and $25 \%$, respectively, with a median survival time (MST) of 4-6 months. Chemotherapy plus irradiation increases survival time not appreciably (2-4 mos.), but is accompanied with heavy side effects. In a retrospective study we achieved a MST of 12 months by an alternative treatment without chemotherapy. This result should be confirmed in a prospective trial.

Methods and Patients: In a prospective open-label trial 46 patients with far advanced, non-resectable, recurrent or metastasized adenocarcinoma of the pancreas were treated. Median age at study entry was 62 years (range 38-82), median Karnofsky index 50\% (range 30-90). Six patients suffered from jaundice and 10 showed ascites at study entry. The multimodal non-toxic treatment consisted of: 1) high-dose enzyme therapy (Pancreon ${ }^{\circledR}$ forte $3 \times 1 /$ die, Wobenzym ${ }^{\circledR} 3 \times 3 /$ die); 2 ) immunotherapy (thymus peptides, mistletoe lectins); 3 ) hormonal therapies (tamoxifen $2 \times 20 \mathrm{mg} / \mathrm{die}, \mathrm{GnRH}$ analogues); 4) differentiating agents (vitamin A-palmitate, 1.25- $\alpha$-hydroxycholecalciferol), and 5) regional deep hyperthermia (13.56 MHz, Theratherm, Italy). Results: MST of the patients. was 10.8 months (range 2.6-77+) since first diagnosis of disease and 4.9 months since start of multimodal treatment. The overall survival rates are shown in the table. Most patients experienced remarkable improvement in quality of life over a long period of time: $68 \%$ were free of pain, $24 \%$ showed marked pain relief; $64 \%$ showed improved appetite, thereof $24 \%$ normal appetite, and reduction of jaundice and ascites. No severe adverse effects of the treatment have been observed.

\section{Overall Survival Rates}

\begin{tabular}{llrlll}
\hline & $\begin{array}{l}\text { 1-year } \\
{[\%]}\end{array}$ & $\begin{array}{l}\text { 2-year } \\
{[\%]}\end{array}$ & $\begin{array}{l}\text { 3-year } \\
{[\%]}\end{array}$ & $\begin{array}{l}\text { 4-year } \\
{[\%]}\end{array}$ & $\begin{array}{l}\text { 5-year } \\
{[\%]}\end{array}$ \\
\hline $\begin{array}{l}\text { From } 1^{\text {st }} \text { Dx } \\
\text { of disease }\end{array}$ & $41 \pm 7$ & $20 \pm 6$ & $13 \pm 5$ & $9 \pm 4$ & $9 \pm 4$ \\
$\begin{array}{l}\text { From alternative Tx } \\
\begin{array}{l}\text { Conventional } \\
\text { therapy (5-FU, GEM) }\end{array}\end{array}$ & $22 \pm 6$ & $9 \pm 4$ & $7 \pm 4$ & $4 \pm 3$ & $4 \pm 3$ \\
\hline
\end{tabular}

Conclusion: In a prospective trial with 46 patients we could confirm previous results of a retrospective analysis of patients with far advanced cancer of the pancreas treated with a complex alternative regimen. Marked improvements in quality of life, pain relief and prolongation of survival compared to conventional chemotherapy could be achieved. Further controlled clinical trials should be accomplished. 


\section{Distant Healing for Common Warts: A Randomised Clinical Trial*}

Harkness, E.; Abbott, N.; Ernst, E.

Department of Complementary Medicine, School of Postgraduate Medicine and Health Studies, University of Exeter, UK

Background and Objective: Distant healing, the alleged treatment transmitted to a distantly located patient, is widely used. Good scientific evidence of efficacy is, however, sparse. This trial was aimed at assessing the efficacy of one form of distant healing on common warts. Methods: A total of 84 patients with warts were randomly assigned either to a group that received 6 weeks of distant healing by one of 10 experienced healers or to a control group that received similar preliminary assessment but no distant healing. Both the patients and the evaluator were fully blinded. Primary outcome measurements were the number of warts and their mean size at the end of the treatment period. Secondary outcome measurements were the change in Hospital Anxiety and Depression Scale and volunteers' subjective experiences. Results: Baseline characteristics were similar between the distant healing $(n=41)$ and control group $(n=43)$. The mean number of size of warts per person did not change significantly during the study. The change in number of warts for the healing group was -0.22 and +1.12 in the control group (difference $=-1.34 ; 95 \%$ confidence interval $=-3.64$ to 0.96 , $\mathrm{p}=0.25$ ). The results relating to wart size also showed no significant inter-group difference. Six patients in the distant healing group and 8 in the control group reported a subjective change for the better. Three people in each group thought that treatment had had some effect on their warts. There was a significant improvement in depression and anxiety in the control group but not the distant healing group. Conclusion: In this setting, distant healing had no significant effect on the number or size of warts.

* This study is in press with the American Journal of Medicine.

\section{Immunological Bystander Reaction of Homeopathy/ Homotoxicology}

Heine, $H$.

Institut für Antihomotoxische Medizin und Grundregulationsforschung, Baden-Baden, Germany

Background and Objectives: Freedmann and Weiner [1] were the first to show that stimulation of $\mathrm{T}$ lymphocytes with antigens in a very low dosage ( $\mu \mathrm{g}$ quantities/kg body weight) induces anti-inflammatory effects by generating regulatory lymphocytes (Th3 cells). They demonstrated that Th 3 cells are capable of synthesizing the anti-inflammatory cytokine-transforming growth factor beta (TGF- $\beta$ ). We tested whether the production of TGF- $\beta$ may be influenced by low-to middlepotentialized substances traditionally used in homeopathy/homotoxicology. Methods: The test system selected comprised whole-blood cultures from 12 healthy donors. 14 different, mainly plant extractions were used at potencies ranging from $1 \mathrm{X}$ through $14 \mathrm{X}$. Incubation lasted for 24 hours. The solvent used in preparing the extracts served as control. After the incubation period the concentration of TGF- $\beta$ was determined by means of a commercially available immunoassay (TGF- $\beta$ ELISA, Hölzel, Germany). TGF- $\beta$ production was calculated as percentages of increase in comparison to the control value. Results: Compared with the control value, all substances were found positive. The highest amounts of TGF- $\beta$ where measured with potencies ranging from $2 \mathrm{X}$ through $8 \mathrm{X}$ (mean value 2.0 -fold of control value). A secondary finding was that the preparations showed individual differences with regard to the TGF- $\beta$ synthesis. Conclusion: Low- to middle-potentialized homeopathic/antihomotoxic preparations seem to intervene in cell language on the cytokine level. The utilized homeopathic preparations given to whole-blood cultures are capable to stimulate the Th3 cells to synthesize the most anti-inflammatory cytokine TGF- $\beta$. This cytokine down-regulates inflammatory leukocytes and therefore plays an essential role in controlling inflammatory processes. This immunological bystander reaction could serve as a model to explain the good effects of homeopathic/antihomotoxic remedies in the treatment of inflammatory as well as chronic inflammatory processes.

\section{Reference:}

Friedmann A, Weiner HL: Proc Natl Acad Sci USA 1994;92:6688-6692.

\section{Clinical Effects and Side Effects of a Lectin-Rich (Iscador Qu Spezial) and a Lectin-Poor (Iscador Pini) Mistletoe Preparation - Results of a Placebo-Controlled Double-Blind Study in Healthy Subjects}

\section{Huber, . $^{1}$; Thoma, D. ${ }^{1}$; Barth, H. $^{2}$; Claßen, K. $^{2}$; Lüdtke, R. $^{3}$;} Werner, $M^{4}$

${ }^{1}$ Center for Complementary Medicine, Department of Gastroenterology, University of Freiburg i. Br.; ${ }^{2}$ Department of Internal Medicine, University of Tübingen; ${ }^{3}$ Institute for Biometrics, University of Tübingen, Germany; ${ }^{4}$ Institute Hiscia, Arlesheim, Switzerland

Background: Mistletoe extracts have been used for decades as a supplementary therapy in cancer patients and are known as well tolerable. Typical side effects are dose dependent inflammatory local reactions (LR) and general symptoms as weakness, cephalgia and subfebrile temperature. A phase-I trial to evaluate the clinical effects and side effects prospectively was not yet performed and was aim of our study. Methods: 48 voluntary healthy subjects participated in the prospective, randomized, placebo controlled double blind study. After check up for health status 16 received placebo, Iscador Pini (IP) or Iscador Quercus spezial (IQ) respectively and applicated it two times a week subcutaneously in increasing dosages for 12 weeks. Weekly clinical controls and laboratory tests (differential blood count, haptoglobin, CRP, less than weekly liver enzymes, electrolytes, creatinine, auto antibodies, mistletoe lectin specific antibodies) were performed. 12 weeks after finishing injections there was a final follow-up. 45 participants finished the study regularly. One participant dropped out for private reasons. Results: Dose-dependent LR occurred in $90-100 \%$ of subjects with maximum dose of either IP or IQ. Also in the placebo group LR occurred, but significantly less frequently. Subjects in IQ group significantly more frequently presented with itching and had a longer delay until onset of LR while the intensity of LR was not significantly different in IQ and IP group. In IQ group LR intensity and delay until onset decreased during study course which correlated to the appearance of mistletoe specific lectin antibodies $(12.5 \%$, in week $4,81.2 \%$, in week $8,81.2 \%$ in week 12 , and $93.7 \%$ in week 26 ) in the peripheral blood. Application of IQ resulted in a significant eosinophilia and increase of haptoglobin. Safety testings showed no abnormality during study course and follow up. No appearence of specific auto antibodies occurred. General side effects showed a wide variety of symptoms which were not severe and could most likely be related to elevated temperature (which was not measured in this study because of its unreliability). Conclusions: IP and IQ induced LR differ in their clinical symptomatology and time course, which can be attributed to different ingrediencies. The particularities of IQ can most likely be related to its high content of mistletoe lectin, especially mistletoe lectin 1 . Severe side effects, allergic reactions, hepato- or nephrotoxicity or development of autoantibodies did not occur during this study. 


\section{Randomised, Placebo-Controlled, Multicentre Trial of Acupuncture for} the Treatment of Chronic Neck Pain*

Irnich, $D^{a}{ }^{a}$; Behrens, ${ }^{b}{ }^{b}$; Molzen, $H_{.}{ }^{c}$; König, A. ${ }^{c}$; Gleditsch, K. $^{1}$; Gleditsch, J. ${ }^{b}$; Stör, $W^{b}$; Krauss, $P^{d}$; Natalis, M. ${ }^{c}$; Senn, E. ${ }^{d}$;

Beyer, A. $^{a}$; Schöps, $P^{b}$

${ }^{a}$ Department of Anaesthesiology, ${ }^{b}$ Department of Physical Medicine and Rehabilitation, ${ }^{\mathrm{C}}$ Institute for Medical Informatics, Klinikum Großhadern, University of Munich, Germany, ${ }^{\mathrm{d}}$ Reha Klinik Bellikon, Switzerland

Objectives: To evaluate the clinical effectiveness of acupuncture for the treatment of chronic neck pain. Methods: 177 patients aged 1885 years with neck pain lasting longer than 1 month participated in this prospective, randomised, placebo-controlled, multicentre trial at 3 universitarian out-patient departments in Germany. The participants were randomised to acupuncture, massage or sham laser acupuncture and were treated five times. Primary outcome measure was motion pain (visual analogue scale, VAS); secondary outcome measures were range of motion (ROM, 3-D ultrasound realtime motion analyser), pressure pain threshold (pressure algometre), assessment of change of spontaneous pain, motion pain and global complaints (seven-point scale), quality of life (MOS SF 36) and a credibility assessment of therapies (VAS). Assessments were performed before, during, 1 week after and 3 months after treatment. Results: One week after treatment the acupuncture group showed significantly greater improvement in motion pain $(\mathrm{p}=0.0052)$ and ROM (score, $\mathrm{p}=0.0001)$ compared to massage, but not compared to sham laser $(\mathrm{p}=0.098, \mathrm{p}=0.151)$. In the subgroup with pain duration $>5$ years $(\mathrm{n}=75)$ the acupuncture group was superior in motion pain compared to massage $(\mathrm{p}=0.0141)$ and sham laser $(\mathrm{p}=0.0313)$. In the subgroup of patients with myofascial pain syndrome $(n=129)$ acupuncture was superior to massage $(\mathrm{p}=0.0012)$ and the $p$ value was 0.0605 compared to sham laser. The acupuncture group showed the best results in nearly all secondary outcome measures. Credibility assessment showed no differences before treatment. No serious adverse events were seen. Conclusions: The results suggest that acupuncture is effective in the treatment of patients with chronic neck pain.

* This work was supported by the Bundesministerium für Bildung und Forschung (BMBF, 01 KT 9406/1). Publication was supported by the Deutsche Ärztegesellschaft für Akupunktur (DÄGfA)

\section{Homeopathic Treatment of Acute Otitis Media in Children -} A Preliminary Randomized Placebo-Controlled Trial

Jacobs, $J^{1}$; Springer, $D .^{2}$; Crothers, $D .^{3}$

${ }^{1}$ University of Washington, Seattle, Washington; ${ }^{2}$ Wallingford Pediatrics, Seattle, Washington; ${ }^{3}$ Evergreen Homeopathic Clinic, Edmonds, Washington, USA

Background and Objectives: The use of antibiotics in the initial treatment of acute otitis media (AOM) is being questioned by many physicians. Homeopathy has been used historically to treat this illness, but there have been no methodologically rigorous trials to determine if there is a positive treatment effect. This pilot study was carried out to determine appropriate outcome measures, sample sizes, and if there is enough evidence of the efficacy of homeopathy in the treatment of AOM to justify a larger trial. Methods: A randomized double-blind placebo control study was carried out in a private outpatient pediatric practice in Seattle, Washington. Seventy-five children aged 18 months to six years with middle ear effusion and ear pain and/or fever for no more than 36 hours were entered into the study. Patients were primarily white and from educated families. Children received either an indi- vidualized homeopathic medicine or a placebo administered orally three times daily for 5 days, or until symptoms subsided. The primary outcome measure was predefined as the number of children referred back to the clinic for treatment failure (fever $>38^{\circ} \mathrm{C}$ and/or any degree of ear pain after 48 hours of treatment, or fever $>39^{\circ} \mathrm{C}$ and/or crying from pain after 24 hours) during the first five days of treatment. Results: There were a total of 12 treatment failures $(30.8 \%)$ in the placebo group compared with $7(19.4 \%)$ in the group receiving homeopathy (p-value $=0.26,95 \%$ CI $0.16,1.78)$. Using these data, a sample size of 243 children in each of two treatment groups would be needed to show a significant difference (alpha $=0.05,80 \%$ power). Analysis of diary symptom scores showed significant differences in the average scores at 24 and 64 hours after treatment in favor of homeopathy $(\mathrm{p}<05)$. Conclusions: These results suggest that there may be a positive treatment effect of homeopathy when compared to placebo in AOM and that a larger study is justified.

\section{Acupuncture in the Treatment of Active Crohn's Disease -} A Randomised Controlled Study

Joos, $S^{1}$; Maluche, $C^{1}$; Maupai, ${ }^{1}{ }^{1}$; Kohnen, R. $^{2}$; Schuppan, $D .{ }^{1}$; Hahn, E.G. ${ }^{1}$ Brinkhaus, B. ${ }^{1}$

${ }^{1}$ Dept. of Complem. Med., Med I, University of Erlangen-Nuremberg, Erlangen; ${ }^{2}$ IMEREM - Inst. for Medical Research Management \& Biometrics, Nuremberg, Germany

Background and Objective: Acupuncture has traditionally been used in the treatment of Crohn's disease (CD) in China and is increasingly applied in Western countries. We investigated whether acupuncture and moxibustion given in addition to conventional therapy is effective treating patients with active CD. Methods: In this randomised, singleblind, controlled trial 51 patients with mild to moderately active $\mathrm{CD}$ were randomly assigned to receive either an acupuncture treatment according to the principles of Traditional Chinese Medicine (TCM group) or a treatment at non-acupuncture points (control group). Both groups were treated 10 times over a time period of 4 weeks while the dosage of the medication was kept constant. The primary outcome measure was change in the Crohn's Disease Activity Index (CDAI) after treatment. Furthermore quality of life assessed by the Inflammatory Bowel Disease Questionnaire, general well-being assessed by Visual Analogue Scale and laboratory parameters were measured. Patients were followed up for 12 weeks. Results: In the TCM group CDAI decreased from $250( \pm 51)$ to $163( \pm 56)$ points $(\mathrm{p}<0.001)$ as compared with a mean decrease from $220( \pm 42)$ to $181( \pm 46)$ points $(\mathrm{p}=0.001)$ in control group after four weeks of acupuncture (TCM vs. control group: $p=0.0008)$. The difference between both groups was still significant after 12 weeks of follow-up $(p=0.027)$. These changes in disease activity were followed by significant improvements in general well-being and in quality of life (TCM vs. control group: $p=0.009$ and $p>0.05$, resp.). In TCM group the clinical improvements were associated with a significant decrease of $\alpha-1$ glykoproteine serum concentration $(\mathrm{p}=0.046)$. Conclusion: Acupuncture is effective in improving symptoms, quality of life and laboratory parameters in patients with active $\mathrm{CD}$. However, acupuncture according to the principles of TCM proved to be significantly more effective than control acupuncture at non-acupuncture points. From these results we conclude that acupuncture provides a useful therapeutic strategy in patients with active $C D$. 


\section{The Test Phase of the BKK-POST on Homoeopathy and Acupuncture:} Designed Feasibilities

\section{Kirschmann, K.; Walach, $H$.}

Department of Environmental Medicine and Hospital Epidemiology, University Hospital of Freiburg i. Br., Germany

Background: At present there is only little knowledge about costs and effects of homoeopathy and acupuncture in general practice. Objective: A comparative evaluation of costs and effectiveness. Methods and Design: A comparative prospective documentation on patients insured for 5 years with the BKK-POST (insurance company) who are provided with free treatment of acupuncture and homoeopathy in Germany. Comparison will be made with patients in conventional treatment. The physicians document the treatment (diagnosis, treatment, duration of treatment). The patients will get generic and specific quality of life questionnaires at the beginning of the treatment, after six months and at the end of treatment. Diagnoses: We investigate patients who suffer from atopic dermatitis, asthma, allergic rhinitis, backache, headache and migraine. Measurement of effectiveness: Measurement of work days lost, hospital stays, costs of rehabilitation and costs of the medicine for all patients. Costs: We evaluate the average costs of the patients pre- and post-treatment who were treated with acupuncture or homoeopathy, and the average costs of a control group who has been treated with a conventional therapy. The insurance company will provide these data. Quality of Life Questionnaires: We measure the general (EuroQol and SF-12) and the specific health status: atopic dermatitis with FLQ-d Version 1.3 b [Augustin et al.,1997], allergic rhinitis with LQ-AR [Mösges], asthma with FAP [Schandry, 1995], backache with FFbH [Kohlmann \& Raspe, 1989] and headache/migraine with MSQ Version 2.1 [Glaxo Wellcome Inc., 1998]. Results: The study is being implemented. We will report the first experience with this design.

\section{Investigation into Non-Analgetic Effects of Acupuncture by fMRI}

Lacour, M. $^{1}$; Gareus, I. $^{2}$; Schulte, A.C. ${ }^{2}$; Zunder, T. $^{1}$; Hennig, J. ${ }^{2}$ ${ }^{1}$ Institute of Environmental Medicine and Hospital Epidemiology, University Hospital, Freiburg i. Br.; ${ }^{2}$ Medical Physics, Department of Radiology, University Hospital, Freiburg i. Br., Germany

Background and Objectives: The mode of action of acupuncture-analgesia has been investigated extensively. In this setting the non-nociceptive acupuncture stimulus is conducted by low-grade or moderategrade myelinated afferent nerve fibres. However little is known about probable non-analgetic effects of acupuncture. The following study investigated the response of functional areas of the human brain to acupuncture of the acupoint Guang Ming which is probably related to vision by fMRI. The investigations were based on the hypothesis that probable non-analgetic effects of acupuncture are also transmitted by the neuronal system and lead to activation of corresponding functional areas e.g. the visual cortex or associated areas. Methods: The fMRIstudy was performed on a 2-T system. The obtained Blood Oxygen Level Dependent (BOLD) signals represent oxygenation-levels of hemoglobin which are increased in activated areas of the brain. In the first study the needles were inserted unilaterally in the acupoint Guang Ming in 7 volunteers without twisting. In the second study the needles were inserted bilaterally into Guang Ming in 17 volunteers with twisting of the needles at one minute intervals to re-enforce the De Qi phenomenon. All volunteers had visual stimulation simultaneously in the first study and in the second study 11 volunteers had visual stimuli. Six volunteers had no visual stimulus during the investigations. The functional maps were obtained by cross-correlation analysis and related to needle insertion and needle twisting. Results: The De Qi phenomenon was triggered in all volunteers and successfully re-enforced in the persons in whom the needles had been twisted. No statistically significant brain activation due to needle insertion was observed. Moderate but significant BOLD signals were detected in response to needle twisting with pronounced effects in the subjects investigated without visual stimulation. In the latters significant BOLD signals were observed in the left and right Gyrus temporalis medialis, the left and right Operculum parietale and the right Insula region. Only visual stimulation produced activation of the visual cortex. Conclusion: The demonstrated activations are probably due to inevitable somatosensory and not to acupuncture-specific stimuli. The probable existence of non-analgetic effects of acupuncture needs to be clarified in further investigations.

\section{Serial Irradiation with Physiological Doses of Ultraviolet Light Improves Antioxidative Capacity of Blood Plasma}

\section{Krause, $R^{1}{ }^{1}$; Lewin, G. $^{2}$; Bühring, $M .^{1}$; Popov, $I^{2}{ }^{2}$; Deuse, $U^{1}$;} Howest, $S^{1}{ }^{1}$; Schildmann, ${ }^{1}{ }^{1}$; Stange, R. $^{1}$; Uehleke, B. ${ }^{1}$

${ }^{1}$ Klinik für Naturheilkunde, Universitätsklinikum Benjamin Franklin der Freien Universität Berlin; ${ }^{2}$ Research Institute for Antioxidant Therapy, Berlin, Germany

Background: Ultraviolet light (UV) is regarded as an oxidative stress from which man should be protected. The authors doubt this general judgement and point to biologically positive effects of UV and the possibility of an adaptation to replied stresses from the environment. Material and Methods: In three randomized controlled trials with altogether 68 persons (trial 1: patients with coronary heart disease, $\mathrm{n}=33$; trial 2: geriatric patients, $n=24$; trial 3: patients with vaginal candidiasis, $n=11) 12$ to 16 irradiations were applied over 4 to 6 weeks in increasing, but strictly suberythematous doses. The spectrum was similar to that of the sun (UVA plus UVB). Patients in control groups were given only UVA in trials 1 and 3, and no treatment in trial 3. Before and after irradiation, plasmatic antioxidative capacity was measured according to the method of photosensitized chemoluminescence as described by Popov and Lewin [Free Rad Biol \& Med 1994;17:267]. Results: Antioxidative capacities increased in the groups with UVB irradiation, while no such effect was seen in the control groups.

\begin{tabular}{|c|c|c|c|c|c|c|c|c|}
\hline & \multicolumn{4}{|c|}{ Verum (UVA plus UVB) } & \multicolumn{4}{|c|}{ Control (UVA or no therapy) } \\
\hline & \multicolumn{2}{|c|}{ before } & \multicolumn{2}{|l|}{ after } & \multicolumn{2}{|c|}{ before } & \multicolumn{2}{|c|}{$\underline{\text { after }}$} \\
\hline & $\mathrm{x}$ & $\mathrm{s}$ & $\mathrm{x}$ & $\mathrm{s}$ & $\mathrm{x}$ & $\mathrm{s}$ & $\mathrm{x}$ & $\mathrm{s}$ \\
\hline 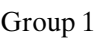 & 569 & 173 & $663^{*}$ & 191 & 583 & 134 & 580 & 137 \\
\hline Group 2 & 696 & 174 & $710 * *$ & 206 & 716 & 225 & 744 & 259 \\
\hline Group 3 & 312 & 51 & 342 & 91 & 312 & 87 & 316 & 78 \\
\hline
\end{tabular}

Unit: $\mu \mathrm{mol} / 1$ ascorbic acid. $* 2 p<0.05 ; * * 2 p<0.01$.

The antioxidative capacity as determined here is measuring integral anti-radical effects of all plasmatic anti-oxidants in an aqueous solution (e. g. uric acid, vitamin C, and proteins). Conclusion: A common principle of natural therapies is confirmed, according to which physiological doses of environmental stresses may improve defense functions. Acute effects of a therapy and those of serial application should clearly be discriminated. There should be no conclusion from short-term damages of single measures to the success of serial therapies. On the contrary, possible noxes may be a therapeutic measure on the long run that lead to favorable reactions of the body. 


\section{Electrostimulation-Acupuncture in the Treatment of Patients with} Cytostatic Drug-Induced Polyneuropathy: A New Concept

Lacour, ${ }^{1}{ }^{1}$; Rostock, $M^{2}{ }^{2}$; Jaroslawski, ${ }^{2}{ }^{2}$; Zunder, $T^{1}$; Güthlin, $C^{1}{ }^{1}$; $\mathrm{Hu}, \mathrm{K}^{3}$; Bartsch, H.H. ${ }^{2}$

${ }^{1}$ Institute of Environmental Medicine and Hospital Epidemiology, University Hospital, Freiburg i.Br.; ${ }^{2}$ Tumor Biology Center, Freiburg i. Br., Germany; ${ }^{3}$ Acupuncture Department, Teaching Hospital of Chengdu University of T.C.M., P.R. China

Background and Objectives: Cytostatic drug-induced polyneuropathy (CDIP) is a common problem in patients receiving high-range chemotherapy (e. g. cisplatine, vincristine or taxol). Although nerve growth factor (NFG) is a promising therapy in preventing CDIP, at present there are no active treatment options. This abstract discusses a new concept for treatment of patients with CDIP by electrostimulation-acupuncture and presents our first clinical experiences. A randomised placebo-controlled study is in progress. Methods: Seven patients with CDIP were treated by electrostimulation-acupuncture. The acupuncture strategy was based on the meridian theory and the course of the peripheral nerves of the extremities. Needles were inserted in Li3, Sp9, Gb41 and Gb34 in the lower extremities and, if symptomatic, additionally in LI4, LI11, SI3 and He3 in the upper extremities. Subsequently we applied a $50-100 \mathrm{~Hz}$ electro stimulation with a composition of squares and sharp waves to the acupuncture-needles in situ. The electrostimulation-acupuncture was applied three times a week over a 2- to 3-week period (6-9 acupuncture treatments per patient). Every patient was asked about his symptoms specific to CDIP at the beginning and the end of the treatment. Therapeutic effects were considered probable if one or more specific symptoms mentioned by the patient improved. Results: Symptoms related to CDIP were par- and hypesthesia, sensory ataxia, reduced fine motoricity, pain, and paralysis of the peroneal nerve. Six of 7 patients showed probable therapeutic effects of electrostimulation-acupuncture. Conclusion: To our knowledge this is the first report on probable therapeutic effects of electrostimulation-acupuncture in patients with CDIP. These effects could be explained by afferent blocking of the somatosensory system due to electrostimulation-acupuncture.

\section{Tablet Quality: A Major Problem in Clinical Trials with} Garlic Supplements

Lawson, L.D.; Wang, Z.J.

Murdock Madaus Schwabe, Inc., Springville, Utah, USA

Background: The failure of five recent clinical trials to show significant reduction in serum cholesterol by non-enteric-coated garlic powder tablets ( 2 brands), four of which used allicin-standardized brand 1, contrasts to many prior positive trials with the same brand. Considerable evidence indicates that allicin, formed enzymatically from alliin, is essential to most of the hypolipidemic effects of garlic. However, efficient allicin formation from tablets requires high tablet alliinase activity, protection of alliinase from gastric acid, and alliinase activation by rapid tablet disintegration at neutral $\mathrm{pH}$. This study was undertaken to determine (1) if impaired release of allicin from these tablets under U.S. Pharmacopeia-defined gastrointestinal dissolution conditions could account for the inconsistency between the trials and (2) if enteric-coated tablets release the expected amounts of allicin. Methods: USP Method $724 \mathrm{~A}$ in vitro drug release test was applied to 10 lots of brand 1, five each of which were manufactured during the years when the trials were positive or negative, as well as to brand 2 and 23 brands of entericcoated tablets. Results: The more recent lots of brand 1, manufactured when the negative trials were conducted, were found to be significantly less resistant to acid-disintegration ( 1.3 vs $2.6 \mathrm{~h}, \mathrm{P}<0.001)$ than the older lots and to release three times less allicin ( 15 vs $44 \%$ of their potential, $\mathrm{P}<0.001)$. Brand 2 released no allicin at all. Most brands of enteric-coated tablets released $<10 \%$ of their allicin claim, due to low tablet alliinase activity and slow buffer disintegration. Breath content of the allicin metabolite, allyl methyl sulfide, was found to correlate well with allicin release determined by USP 724 A. Conclusions: Ineffective allicin release is a serious problem for most brands of garlic powder tablets and is centered on alliinase activity rather than alliin content. This may well account for much of the discrepancy found in the clinical trial outcomes. Clinical trials employing garlic supplements should make sure the allicin release has been determined under USP-standardized drug release conditions.

\section{Systemic Enzyme Therapy in Oncology}

\section{Leipner, J.; Saller, $R$}

Department of Natural Medicine, University Hospital, Zurich, Switzerland

Plant extracts with a high content of proteolytic enzymes have been used for a long time in traditional medicine. Its therapeutic use is partly based on scientific studies and partly empirical. The aim of the current paper is to provide an overview of clinical trials of systemic enzyme therapy in oncology, and to discuss the evidence for possible mechanisms of action.

The literature search was based on Medline (1966-1999), EMBASE (1980-1999), and AMED (Allied and Alternative Medicine, 19851999) databases. In addition, the firm MUCOS (Geretsried, Germany) allowed us to examine hitherto unpublished studies.

Clinical studies of the use of proteolytic enzymes in oncology have been carried out on an enzyme preparation consisting of a combination of papain, trypsin and chymotrypsin (Wobe-Mugos ${ }^{\circledR}$ ). In seven prospective clinical studies with 692 patients, oral enzyme therapy has generally been found to be a well-tolerated form of treatment for the relief of side-effects caused by other tumour therapies (chemotherapy and radiotherapy) and for improving the quality of life.

The precise mechanism of action of systemic enzyme therapy, however, still remains an open question. The ratio of proteinases to antiproteinases, which is increasingly being used as a prognostic marker in oncology, appears to be influenced by the oral administration of proteolytic enzymes, probably via an induction of the synthesis of antiproteinases. There are numerous alterations of the cytokine composition during therapy with orally administrated enzymes. However, whether the induction of antiproteinases has a direct or indirect effect on cytokine metabolism is a question that cannot yet be answered.

In conclusion, the use of proteolytic enzymes in oncology has shown that enzyme therapy can reduce the side-effects caused by radiotherapy and chemotherapy. There is also evidence that, in some types of tumours, survival may be prolonged. Nevertheless, the number of clinical studies is too limited to give a final judgement of the efficacy of systemic enzyme therapy although this therapy is well accepted as an evidenced-based therapy. 


\section{A Double-Blind, Randomised, Controlled Clinical Trial of Ultramolecular Potencies of House Dust Mite in Asthmatic Patients}

Lewith, G.T.

School of Medicine, University of Southampton, UK

Introduction: Based on Reilly's pilot work in Glasgow, we carried out a large-scale, double-blind, randomised, placebo-controlled trial of homoeopathic immunotherapy (HIT) for asthma using a homoeopathic $30 \mathrm{C}$ dilution of house dust mite extract versus an identical placebo. Homoeopathy predicts an initial deterioration in outcome followed by an improvement. Methods: Patients were recruited and seen in 38 general practices in Hampshire and Dorset over a 2-year period (96-98). The medication and placebo were prepared by Boiron Laboratories. After a 4-week baseline assessment, asthmatic patients who were house dust mite sensitive (skin prick positive) were randomised to receive HIT or placebo in a single split dose over 24 hours. They were then assessed over a 16-week period, with three clinic visits and diary outcome assessment every other week. Clinic based assessments were FEV1, quality of life (QOL), and mood. Diary based assessments were morning and evening peak expiratory flow (PEF), a visual analogue scale (VAS) of asthma severity, diary QOL, and daily mood. A variety of statistical analyses were used. We chose the most rigorous; a repeated measures Analysis of Covariance, to test whether there was a difference in change between the two treatments over the post-randomisation assessment period. Results: Two hundred and forty-two patients were randomised and 202 completed the study. Three variables were significant: Morning PEF, $\mathrm{p}=0.025$; VAS, $\mathrm{p}=0.017$; Mood, $\mathrm{p}=0.035$, and in each case there was a nonmonotonic interaction between treatment condition and week of assessment with two maxima. Baseline measures were equivalent, blinding was effective, and improvement was independent of belief in complementary medicine. Conclusion: This rigorous study clearly supports the hypothesis that ultramolecular dilutions can be differentiated from placebo using HIT as an experimental model.

\section{The Methodological Quality of Randomized Clinical Trials of Homeopathy, Herbal Medicines, and Acupuncture}

Linde, K. ${ }^{1,3}$; Jonas, W.B. ${ }^{2}$; Melchart, D. ${ }^{1}$; Willich, S. $^{3}$

${ }^{1}$ Centre for Complementary Medicine Research, Dpt. Internal Medicine II, Technische Universität, München, Germany; ${ }^{2}$ UHUHS, Bethesda, USA; ${ }^{3}$ Humboldt University, Berlin, Germany

Objectives: To investigate the methodological quality of randomized clinical trials of homeopathy, herbal medicines and acupuncture and to compare it with the quality of trials in conventional medicine. Methods: 207 randomized and quasi-randomized clinical trials on humans treated for curative, palliative or preventative purposes from five previously published systematic reviews on homeopathy (1 review), herbal extracts (2) and acupuncture (2) were included in this analysis. Methodological quality was assessed using single-quality items (randomization, blinding, reporting and handling of drop-out and withdrawals) and a validated scale (the Jadad scale). The results were compared to those of two similar published analyses on conventional medicine trials. Results: While the quality of the complementary medicine trials was highly variable the majority had important shortcomings in reporting and/or methodology. Major problems in most trials were in the description of the method to conceal allocation and the handling of drop-outs and withdrawals. There were relevant differences in single quality components between the different complementary therapies: For example, homeopathy trials were less often explicitely randomized, acupuncture trials reported less often an adequate method to conceal random allo- cation, and trials on herbal extracts had better summary scores. Trials published in journals listed in Medline, published more recently, and published in English language scored significantly higher than trials not meeting these criteria. The Jadad score of the complementary medicine trials reviewed in this study was very similar to that from two major published analyses of trials on conventional medicine. Conclusions: Trials of complementary therapies often have relevant methodological shortcomings. However, according to a validated and widely used scale their quality of reporting is not worse than that of trials on conventional medicine.

\section{Should Systematic Reviews Include Non-Randomized Trials? Quality and Results of Various Study Types Investigating the Effect of} Acupuncture on Idiopathic Headaches

Linde, K. ${ }^{1,2}$; Melchart, D. ${ }^{1}$; Scholz, M. ${ }^{3}$; Willich, S. $^{2}$

${ }^{1}$ Centre for Complementary Medicine Research, Dept. Internal Medicine II, Technische Universität Munich; ${ }^{2}$ Humboldt University Berlin; ${ }^{3}$ Technische Universität Munich, Germany

Background and Objective: Most systematic reviews of treatment interventions exclude non-randomized trials. Although non-randomized trials cannot provide good evidence for effectiveness the inclusion of such trials in a systematic review might provide useful information on prognosis, especially in case of chronic diseases and if evidence from randomized trials is insufficient or lacking. We aimed to investigate to what extent characteristics, quality and results of different study types on acupuncture treatment for idiopathic headaches differ. Methods: We tried to identify all studies reporting clinical outcome data for acupuncture treatment for idopathic headaches. Descriptive characteristics, quality aspects and proportions of patients improving after treatment were extracted. Summary estimates were calculated for different study types and studies meeting predefined quality criteria. Results: A total of 59 studies was identified: 24 randomized trials, 5 non-randomized controlled trials, 10 uncontrolled prospective trials, 10 case series, and 10 cross-sectional studies. Randomized trials and prospective uncontrolled studies had significantly better quality than the other studies but both categories contained studies of completely insufficient quality. Proportions of patients reporting an improvement after acupuncture were significantly lower in randomized, prospective uncontrolled trials, and cross-sectional studies. Conclusions: In the study set investigated the quality of non-randomized controlled trials and case series was in average so low that it would not seem advisable to include such studies in a systematic review. Some prospective uncontrolled trials provided potentially relevant information on long-term prognosis which could complement data from RCTs.

\section{Developing Guidelines for EU and National Policy Makers}

Long, A. ${ }^{1}$; Connolly, $S^{2}$

${ }^{1}$ University of Salford, Salford, UK; ${ }^{2}$ European Shiatsu Federation, Dublin, Ireland

Background and Objectives: Concurrent with the widespread use of complementary and alternative medicine (CAM) is a public demand and right for guarantees of safety and effectiveness. While each discipline is keen to follow a route of self-regulation and develop their own codes of ethics and professional practice, national and European Union policy makers need guidance on ways to assess the quality of CAM professional practices, safety and benefits, ... with a view to their legal recognition (European Parliament 1999). The study aims to bridge this gap in knowledge. It aims to develop a framework of key areas of as- 
sessment and evaluation and to pilot the framework within the one CAM discipline of shiatsu. Methods: The framework is being derived in two main ways. The first involves drawing together a summary of, inter alia, the principles and practices that differentiate CAM from conventional medicine, their alternative and/or complementary nature, the notions of holism, treatment and healing and associated the legal models to which CAMs may be subject in different EU countries. This will be gleaned from the literature and consultation with practitioner and academic experts. The second comprises a set of key questions to ask about the state of knowledge about the safety and effectiveness of $\mathrm{CAM}$, and potential key data sources. The framework will then be piloted within shiatsu, prior to wider testing within other CAM disciplines. Results: The paper will present the key components of the developing framework. Conclusions: If a sound framework that is acceptable to CAM professions, practitioners and policy makers can be developed, this will enhance the credibility of CAM within conventional medicine and be of wider assurance to therapy users and citizens in general. It will also promote the use of CAM alongside conventional medicine.

\section{Reference:}

European Parliament (May 1999) Resolution on the Status of Non-Conventional Medicine

\section{Current Use of Alternative Therapies in Physiotherapy: A Questionnaire Survey}

\section{McNeill, S.; Walsh, D.M.; Baxter, G.D.}

Rehabilitation Sciences Research Group, University of Ulster, Jordanstown, N. Ireland

Background: There is little objective data on the use of alternative therapies; following a recent retrospective study of such use [McNeill et al. 1998], it was decided to complete a survey of Chartered Physiotherapists in Southern Ireland. Objectives: The main aims of this survey were to establish the level of current use of alternative therapies by physiotherapists and additionally training received; an additional aim was to identify those painful conditions which responded best to alternative therapies. Methods: A random sample $(n=100)$ of members of the Irish Society of Chartered Physiotherapists (ISCP) was surveyed by post. Data were analysed using Statistical Package for Social Sciences (SPSS). Results: A response rate of $82 \%$ was obtained. Results identified details of clinical grading, public and private practice, training in alternative therapies, conditions which responded best, together with patient suitability criteria. Physiotherapists who had relevant postgraduate training $(15.9 \%)$ had trained in a variety of therapies including acupuncture, shiatsu, sports massage, cranio-sacral therapy and trigger point therapy. Of those using alternative modalities to treat painful conditions, $19 \%$ found them 'effective' or 'very effective' in the management of low back pain, $35 \%$ found them 'effective' or 'very effective' in the management of cervical pain. Alternative therapies were found to be 'effective' or 'very effective' in $20 \%$ of chronic soft tissue problems. The criteria for using alternative therapies in musculoskeletal problems included lack of response to 'non physical' treatments $(13.4 \%)$, or other types of physiotherapy (35.4\%). The main reasons for not having undertaken formal training were cost $(34.1 \%)$ and lack of time $(52.4 \%)$. Conclusion: This survey has identified that $15.9 \%$ of respondents were practising or had trained in alternative therapies. This confirms (for the first time) the level and widespread use of alternative therapies in Ireland by Chartered Physiotherapists.

\section{Reference:}

McNeill S, Walsh DM, Baxter GD: Acupuncture and physiotherapy: A preliminary retrospective study of the scope of practice. Physiotherapy Ireland 1998;19:19-25.

\section{Acupuncture versus Midazolam for Gastroscopy - A Comprehensive} Cohort Study

Melchart, D. ${ }^{1}$; Steger, H.G. ${ }^{2}$; Linde, K. ${ }^{1}$; Makarian, K. ${ }^{2}$; Hatahet, Z. ${ }^{2}$; Brenke, $R^{2}$; Saller, $R^{3}$

${ }^{1}$ Research Centre for Complementary Medicine, Department of Internal Medicine II, Technische Universität, Munich; ${ }^{2}$ District Hospital Simbach, Germany; ${ }^{3}$ University of Zurich, Switzerland

Objective: To investigate whether acupuncture is an effective and desired pretreatment for gastroscopy in comparison with midazolam. Methods: This trial was a comprehensive cohort study on 108 in-patients of a district hospital in southern Germany undergoing gastroscopy for diagnostic purposes. Patients having given consent were allocated randomly while patients who had a preference received the treatment of their choice. Main outcome measures were the patient's overall assessment on a visual analogue scale (VAS) and the number of patients who would choose the same pretreatment for the next gastroscopy. Results: Twenty-eight patients (26\%) agreed to be randomized, $21(20 \%)$ chose acupuncture, 51 (47\%) midazolam, and 8 (7\%) did not want pretreatment. Patients receiving midazolam rated the examination as slightly less troublesome than those receiving acupuncture (VAS mean difference $9 \mathrm{~mm}, 95 \%$ confidence interval -2 to $20 \mathrm{~mm}$, $\mathrm{p}=0.12) .70 \%$ of patients treated by acupuncture and $85 \%$ treated by midazolam would choose the same pretreatment for a further gastroscopy (rate ratio $0.82,95 \%$ CI 0.64 to 1.06 ). $\mathrm{O}_{2}$ saturation, blood pressure and heart rate were significantly lower in patients receiving midazolam. Characteristics and outcomes of randomized and non-randomized patients did not differ significantly. Conclusions: The results of this trial suggest that while acupuncture seems, in principle, to be a feasible and satisfactory pretreatment option for gastroscopy, sedation is preferred by the majority of patients. Acupuncture might be more valuable for out-patients in whom the duration of sedative effects of midazolam is more problematic.

Fasting-Induced Mood Enhancement - Metabolic and Neuroendocrine Interactions: Effects of Therapeutic Fasting on Urinary Catecholamine and Cortisol Excretion, Cytokine Production, Heart Rate Variability and Mood in Patients with Chronic Pain Syndromes

\section{Michalsen, $A^{1}{ }^{1}$; Schumann, R.R. ${ }^{2}$; Janert, $S^{1}{ }^{1}$; Huether, ${ }^{3}{ }^{3}$,} Melchart, D. ${ }^{4}$ Dobos, G. $^{1}$

${ }^{1}$ Department of Internal and Integrative Medicine, Hospital EssenMitte; ${ }^{2}$ Institut für Mikrobiologie, Charite Medical Centre, Humboldt University Berlin; ${ }^{3}$ Psychiatric Hospital, University Göttingen; ${ }^{4}$ Münchener Modell, Centre for Complementary Medicine Research, München, Germany

Introduction: Fasting has been successfully used as a therapy in 'traditional German naturopathic medicine' for chronic pain disorders and metabolic syndrome. Short periods of fasting followed by vegetarian diets can cause clinically relevant long-term improvement in patients with rheumatoid arthritis and chronic pain syndromes of the locomotor system, which was shown recently in randomised controlled trials. Yet the immunological and autonomic effects of fasting are not fully understood. Moreover, fasting has a long-standing tradition and is still practised as a general health-promoting and mentally stimulating ritual in various religious systems. Systematic documentation of these effects has not been done so far. Against this background we were interested to record the course of mood and emotional well-being in patients during fasting and to document, in a parallel manner, heart rate variability, urinary catecholamine and cortisol excretion, and cytokine production. 
Patients and Methods: 28 consecutive patients in a specialised clinic with chronic pain syndromes or rheumatoid arthritis were included. 22 patients practised fasting over 7 days as described by the method of O. Buchinger, 6 patients received only standard treatment and served as controls. Accompanying scales and questionnaires included visual analog scales for general mood and main complaints, the 'Basler Befindlichkeits-Skala' and the 'Profile of mood states (POMS)'. Standardised short-term recordings of heart rate variability for spectral analysis were done using a commercial Holter ECG system. Urine for catecholamine and cortisol analysis was collected during 19:00 p.m. and 8:00 a. m., serum probes were analysed for measurement of TNF-alpha, IL-1, 2, 6, 8. Results: Mean age was 45 years, and all subjects were of normal weight (body mass index between 20 and $25 \mathrm{~kg} / \mathrm{m}^{2}$ ). A mean weight loss for fasting patients of $2.9 \mathrm{~kg}$ (SD $0.3 \mathrm{~kg}$ ) was found until day 11. A constant and significant $(p=0.05)$ increase of nightly epinephrine and norepinephrine excretion and a slight increase in nightly cortisol excretion with a maximum on the seventh fasting day was found in fasting patients vs. controls. Fasting-induced mood enhancement was correlated with catecholamine excretion. Spectral analysis of heart rate variability showed a significant increase of mainly sympathetic controlled low-frequency power (LF) and a slight increase of high-frequency power (HF) and total power. For LF/HF ratio a nonsignificant decrease over the observational period was found. Kinetics of cytokine levels reflected fasting-induced immunomodulation. Conclusions: Fasting leads to a significant increase in mood, reaching baseline levels again after termination of fasting. Mood enhancement correlates with an increase in urinary catecholamine excretion, but not cortisol excretion. HRV demonstrates a significant increase in lowfrequency power, but also slight increases in vagally dominated highfrequency power and total power. Our findings provide evidence for the psycho-neuro-immunological basics of fasting and the well-observed clinical effects.

\section{Ethical Approval of Research on Complementary Medicine}

\section{Milton, $G$.}

School of Nursing, Monash University, Peninsula Campus, Frankston, Victoria, Australia

The increased use of Complementary medicine in the community has rightly led to an obligation by health care providers for a corresponding increase in evidenced-based research in relation to their use. The term Complementary medicine includes a very diverse group of therapies, and it is well known that some are potentially more harmful than others. Furthermore, many may have little or no benefit, whereas others may prove to be 'medical breakthroughs' in terms of treatment. Those researchers who wish to study the effects of a treatment on humans, including Complementary medicines, generally require ethical approval before commencing their project.

Understanding the ethical approval process is essential to facilitate research. The aim of this paper is to highlight a variety of ethical issues that are likely to influence Ethics Committee approval in relation to Complementary medicine research. For example, some questions that have arisen in my experience on an Ethics Committee will be addressed, including: Should the study of aromatherapy in hospitals be considered a drug trial, or is it only a perfume? What if approval had been given for research of a herbal remedy that was later found to be banned? Who would be held accountable if a serious unexpected harm occurred as a result of a research project involving Complementary medicine?

In relation to informed consent, how much information can be given about a therapy if little or no studies have been done in the past? To what extent should the Ethics Committee insist on evidence of efficacy and potential harms, and how confident must the researcher be that the potential harms of any therapy have been identified before commencing the study?

\section{Sickness Absence in Chronically ill Patients Treated with Complementary Medicine}

Moebus, S.; Lehmann, N.; Jöckel, K.H.

Institute for Medical Informatics, Biometry and Epidemiology, Essen, Germany

Background and Objectives: The purpose of this ongoing outcome study is to investigate the effects of an array of Complementary Medicine interventions on chronic diseases using predefined health indicators. The study, including more than 900 volunteers, is sponsored by 22 German company health insurances. In this contribution we analyze observed trends in sick leave, matched by covariables like age, gender, diagnosis and health improvement. Methods: Sick leave data of 320 patients at work were sampled over a period of 10 years, retrospectively and prospectively. We test trends in the subcohort $(\mathrm{n}=170)$ with nonmissing sick leave data in the observation period ranging 3 years before and after inclusion. Nonparametric tests - Friedmann test, rank sum test for dependent samples, repeated measures rank variance analysis are used. The cumulative sick leave incidence (proportion of patients with any sick leave per year) serves as indicator for the whole study group. In order to control for secular trends we standardize on year, age and gender, based on the working population of all German company health insurances. Results: Sickness absence days per year reveal a slow, nonsignificant increase from 23 (95\% CI 16-30) to 29 (CI $23-$ 36) within 5 years until inclusion, followed by a pronounced significant decrease $(\mathrm{p}<0.01)$ from 28 (CI 20-35) in the first year to 20 (CI 14-27) days in the second year of treatment, sustaining for the next 2 years. Sick leave cases and durations per case show the same type of time dependence. In the retrospective period the cumulative sick leave incidence per year varies only between $62 \%$ and $65 \%$. It decreases significantly during and after treatment, 4 years after inclusion down to $50 \%$ (CI $42 \%-59 \%$ ). These results provide a strong argument against possible regression-to-the-mean artefacts. Conclusion: For the first time a profound analysis of nonconventional medicine effects on sick leave can be presented. There is surprisingly clear evidence that the intervention reduces sick leave in chronically ill patients in this outcome study. These observations are corroborated by data on the improvement of the patients' health status.

\section{A Systematic Review of Clinical Studies on Fasting and Egetarian Diets in the Treatment of Rheumatoid Arthritis}

Müller, H. ${ }^{1}$; Wilhelmi de Toledo, $F^{2}$; Resch, K.L. ${ }^{1}$

${ }^{1}$ Forschungsinstitut für Balneologie und Kurortwissenschaft, Bad Elster; ${ }^{2}$ Klinik Buchinger, Überlingen, Germany

Background and Objectives: The pharmacological treatment of rheumatoid arthritis (RA) is often accompanied by marked side-effects. The idea that fasting followed by vegetarian diet may be a reasonable treatment alternative is supported by patient impressions, clinical experience, and some scientific studies $[1,2]$. The objective of our investigation was to review the empirical evidence concerning this therapeutic strategy. Methods: Articles on fasting in RA were identified by a search of MEDLINE and other databases, by references in articles, and by consultation of experts. The results of controlled studies which reported follow-up data for a period of at least three months after fasting were combined by means of a meta-analysis. Results: Thirty-one articles re- 
ported original studies on fasting in RA. There is general agreement and conclusive empirical evidence on the immediate anti-inflammatory action of fasting in RA. With re-introduction of food, however, symptoms usually come back. Whether beneficial effects last with sustained vegetarian diets is less certain, since only four controlled studies with different methodological quality fulfilled the above mentioned followup criterion. The meta-analysis of these studies points at significant long-term improvements in pain related outcome measures $(\mathrm{p}<0.01)$. Conclusion: Without any reasonable doubt, fasting ameliorates RA symptoms very impressively in the short term. The long-term effects in combination with subsequent vegetarian diets are poorly investigated. Although the available evidence supports the notion that this complementary treatment approach may cause important improvements in RA, more well-conducted long-term studies are needed to convincingly confirm the efficacy of this treatment option.

\section{References:}

1 Fahrner H: Fasten als Therapie. Stuttgart, 1985, Hippokrates.

2 Kjeldsen-Kragh J, Haugen M, Borchgrevink CF, Laerum E, Eek M, Mowinkel $\mathrm{P}$, et al.: Controlled trial of fasting and one-year vegetarian diet in rheumatoid arthritis. Lancet 1991:338:899-902.

\section{Rheumatology Nurses' Beliefs towards Complementary Medicine with a Focus on Aromatherapy}

Osborn, C.E. ${ }^{1}$; Barlas, $P^{1}$; Barlow, J.H. ${ }^{2}$; Baxter, G.D. ${ }^{3}$

${ }^{1}$ Physiotherapy Subject Group; ${ }^{2}$ Psychosocial Rheumatology Research Centre, Coventry University, England, ${ }^{3}$ University of Ulster, Northern Ireland

Objectives: A significant proportion of people with rheumatic diseases have used complementary medicine to supplement their conventional treatment. However, investigations of the beliefs towards, and use of, complementary medicine among rheumatology nurses are limited, particularly for aromatherapy. Therefore, this study aimed to explore the current trends in this area within rheumatology nursing. Methods: Selfadministered questionnaires $(\mathrm{n}=252)$ were distributed by post to rheumatology nurses throughout Britain. The questionnaire was designed to provide descriptive information on both rheumatology nurses' beliefs towards, and use of, complementary medicine. The advice they provided to patients was also investigated. Following two mailings, the non-responders $(n=60)$ were followed up. Data were analysed using the Statistical Package for the Social Sciences. Results: The overall response rate was $76.2 \%(\mathrm{n}=192)$ with $62.3 \%(\mathrm{n}=157)$ respondents currently working in rheumatology. Complementary medicine was practised by $9.2 \%(n=13)$ of the nurses. Interestingly, the complementary medicine advice provided by the nurses was significantly correlated with their personal experience of receiving treatments $(\mathrm{p}=0.0013)$. Patients' private use of complementary medicine was reported by $79.3 \%$ of the nurses; acupuncture and aromatherapy being the most popular. Rheumatology nurses believed that symptoms of rheumatic diseases could be helped by complementary medicine in general $(85.1 \%)$ and specifically aromatherapy (57.9\%). Regarding aromatherapy, over half the respondents believed that aromatherapy provided more than relaxation and had some therapeutic effect on pain. Conclusions: The current study suggests that personal experience of complementary medicine by rheumatology nurses greatly influenced the advice given to patients. In addition, communication between patients and the nurses regarding complementary medicine was evident. These findings highlight the importance of further research in this area. Future studies will investigate the practice and beliefs of rheumatologists and people with rheumatic diseases towards complementary medicine, with a focus on aromatherapy.
The Efficacy of Ginkgo biloba Extract for Intermittent Claudication. A Meta-Analysis of Randomized Clinical Trials

Pittler, M.H.; Ernst, E.

Department of Complementary Medicine, School of Postgraduate Medicine and Health Sciences, University of Exeter, UK

Background and Objectives: The optimal treatment of intermittent claudication has not yet been identified. Ginkgo biloba extract is one conservative option reported to have beneficial effects. This paper provides a meta-analysis of the efficacy of Ginkgo biloba extract for intermittent claudication assessing randomized, placebo-controlled, double-blind trials. Methods: Literature searches were performed to identify all studies on the subject. Computerized databases were Medline, Embase, Biosis, AMED, CISCOM and the Cochrane Library. Manufacturers of commercial Ginkgo biloba products and authors of original publications and reviews were contacted to provide additional information. No language restrictions were imposed. Results: Eight randomized, placebo-controlled, double-blind trials were included. A meta-analysis of all trials shows a significant difference in the increase of pain-free walking distance in favor of Ginkgo biloba (weighted mean difference: 34 metres, $95 \%$ confidence interval: 26-43). In studies using similar methodological features (ergometer speed: $3 \mathrm{~km} / \mathrm{h}$, inclination: $12 \%$ ) this difference was 33 metres in favor of Ginkgo biloba (95\% confidence interval: 22-43). Adverse effects were rare, mild and transient. Conclusions: Collectively these data suggest that Ginkgo biloba extract is superior to placebo in the symptomatic treatment of intermittent claudication. However, the size of the overall treatment effect is modest and therefore seems of uncertain clinical relevance.

\section{The Efficacy of Kava Extract for Anxiety. A Systematic Review and Meta-Analysis}

Pittler, M.H.; Ernst, E.

Department of Complementary Medicine, School of Postgraduate Medicine and Health Sciences, University of Exeter, Exeter, UK

Background and Objectives: Synthetic anxiolytic drugs are effective for anxiety, but are burdened with serious adverse effect. Constraints on resources and time often render therapies such as psychological interventions impracticable. Thus, an effective oral medication with few adverse effects would be a welcome addition to the therapeutic repertoire. This systematic review and meta-analysis is aimed at assessing the evidence for or against the efficacy of kava extract as a symptomatic treatment for anxiety. Methods: Systematic literature searches were performed in the computerized databases Medline, Embase, Biosis, AMED, CISCOM and the Cochrane Library (all from their respective inception to June 1998). The search terms used were kava, kawa, kavain, Piper methysticum and Rauschpfeffer (German term for Piper methysticum). Experts on the subject were contacted to provide further information. There were no restrictions regarding the language of publication. Double-blind, randomized, placebo-controlled trials of oral kava extract for the treatment of anxiety were included. All publications were blinded prior to assessment by a person not involved in the study. Data were extracted in a standardised, predefined fashion independently by the two reviewers. Methodological quality of all trials was assessed. Results: Fourteen double-blind RCTs were retrieved. Seven were placebo-controlled and all suggest superiority of kava extract over placebo. The meta-analysis of three trials suggests a significant difference in the reduction of the total score on the Hamilton Anxiety Scale in favor of kava extract (weighted mean difference: 9.69, 95\% confidence interval: $3.54-15.83$ ). Conclusion: These data imply that kava ex- 
tract is superior to placebo as a symptomatic treatment for anxiety. Kava extract is therefore a herbal treatment option for anxiety worthy of consideration.

\section{Role of Intentionality and Imagery in Mental Healing}

Rein, $G$.

John F Kennedy University, Northport, NY, USA

Background: Conscious intention and mental imagery are important aspects of complementary medicine, whether it be the intention of the mental healing practitioner or the imagery used by patients imaging their disease. Most mental healing studies have focused on the phenomenological aspects and have not examined the role of intention or imagery. Methods: In vitro methods were used to study the efficacy of a mental healing practitioner by measuring the growth of tumor cells as the amount of radioactive thymidine incorporated into DNA relative to the total number of cells. Three dishes were treated by a healer and three control dishes were treated simultaneously by a non-healer while reading a book. Five different mental intentions were studied in five separate experiments. Additional experiments were conducted with one intention being held constant, but the mental imagery used varied. A third party then brought all six dishes back to the laboratory where they were blindly labelled. The dishes were then treated with radioactive thymidine and measured after 24 hours of growth. Results were calculated as percent change relative to controls. Results: Results indicate three of the intentions showed a statistically significant decrease in cell growth. The intention to return the tumor cells to the natural order and harmony associated with healthy cells was the most effective, producing a $39 \%$ inhibition of cell growth. The efficacy of the different intentions varied as much as two-fold. Thus, certain intentions were more efficacious than others, whereas other intentions appeared to have no effect on tumor growth. Using the intention to circulate energy through the practitioner's body in a specific manner, it was observed that imaging very few cells in the Petri dish produced an $18 \%$ inhibition of growth, whereas imaging a larger number of cells produced a $15 \%$ increase in cell growth. Conclusion: The results confirm the validity of this methodological approach for studying the role of intention and imagery in mental healing. In future studies, this approach could also be used to measure target specificity and the efficacy of different intentions/imagery for different practitioners.

\section{Discrimination of the Organic, Functional and Psychic Origin of the Disorders Treated with Acupuncture. A Survey among Psychiatrists and General Practitioners}

Romoli, M.; Fiorio, R.; Rogora, G.A.

Società Italiana di Riflessoterapia, Agopuntura, Auricoloterapia S.I.R.A.A., Italy

Background and Objectives: Chinese acupuncture often uses concepts and terms that Western doctors must be able to translate and use before they can put them into practice. The symptoms described in Chinese acupuncture textbooks are partially different from those used in our academic medicine and may cause some confusion. For example, 'weakness of spleen and stomach' may be considered as a dysfunction of the two organs or may be correlated to an emotional condition like 'worry and dissatisfaction'. The aim of this survey was to clarify whether the symptoms reported in two Chinese textbooks were rated by Western doctors as organic, functional (psychosomatic) or psychic. Methods: Two lists of symptoms, referable to the disorders treated by the acupoints of the 14 meridians and by 'extra' points, were taken from 2 Chinese textbooks. The two lists were sent by mail/e-mail to 106 doctors, 40 psychiatrists (PS) and 66 general practitioners (GPs). Some doctors practiced acupuncture besides their principal profession and were considered as a subgroup. Doctors in this category were included in the sample if they had practiced acupuncture for at least three years. The colleagues were asked to rate each symptom as organic, functional or psychic/psychiatric. Responses were obtained from 35 PS (with a response rate of $87.5 \%$ ), of which 8 were acupuncturists and 27 were not, and from 46 GPs (response rate $70 \%$ ), of which 21 were acupuncturists and 25 were not. Results: a) Symptoms in text A were rated as $72.1 \%$ organic, $22.1 \%$ psychosomatic and $5.8 \%$ psychic by PS respondents, and $69.3 \%, 26.3 \%$, and $4.4 \%$, respectively, by GPs. Responses on text B symptoms were, respectively, $75 \%, 21.2 \%$, and $3.8 \%$ for the PS, and $70.2 \%, 27.3 \%$ and $2.5 \%$ for the GPs. b) Of the symptoms listed in text A and classified by at least one respondent as psychic, $41.4 \%$ were for the PS and 28.2 for the GPs. As for text B, the percentages were $34 \%$ and $30.8 \%$, respectively. Of the symptoms on which we found agreement of at least $50 \%$ of the respondents within each different group of doctors, the following were considered as psychic in text A: hysteria, schizophrenia, mental disorders, mania, anorexia, anxiety, neurasthenia, neurosis, insomnia, sleep disorders; in text B: mania, depression, anorexia, irritability, insomnia, globus hystericus. Conclusions: This survey shows that the Chinese textbooks we have analysed report mostly symptoms Western doctors consider as organic. There are no significant differences in the rating of symptoms among the different groups and subgroups. It has to be stressed that in the Chinese textbooks psychic symptoms are described very scantily. For example, text A does not mention depression, and text B instead does not include anxiety. The reason of these omissions is unclear and could be due to one or more of the following factors: 1) The frequency of psychic disorders in China is low and does not draw acupuncturists' attention, 2) traditional Chinese medicine does not believe acupuncture to be effective in the treatment of these disorders, 3) anxiety and depression belong, according to the Chinese, to more general syndromes including organic, psychosomatic and psychic symptoms. Future research should be focused to explore these aspects.

\section{Pharmacokinetics of Alkamides and Cichoric Acid from Echinacea purpurea}

Rostock, M. ${ }^{1}$; Osowski, ${ }^{1}{ }^{1}$; Elsässer-Beile, U. ${ }^{2}$; Häring, B. ${ }^{1}$; Hauns, B. ${ }^{1}$; Kuhlmann, J. ${ }^{1}$; Maier-Lenz, H. ${ }^{1}$; Massing, U. ${ }^{1}$; Schmidt, S. ${ }^{1}$; Schulz, K. ${ }^{3}$; Schumacher, M. $^{3}$; Bartsch, H.H. ${ }^{1}$

${ }^{1}$ Tumor Biology Center at the Albert-Ludwigs-University Freiburg; ${ }^{2}$ Institute of Immunobiology and ${ }^{3}$ Institute of Medical Biometry and Medical Informatics at Albert-Ludwigs-University Freiburg, Germany

Background and Objectives: Pharmaceutical preparations of Echinacea purpurea (E.p.) are among the most popular herbal products. Among other constituents of E.p., Dodeca-2E,4E,8Z,10E/Z-tetraenoic acid isobutylamide (alkamides-8,9) and 2,3-Di-0-caffeoyl-tartrate (cichoric acid) are described as immunomodulating substances. To investigate the pharmacokinetics of both substances in men, a phase-1 study (prospective, open, non-randomised) was performed. Methods: $10-30-60$ 90-120 ml of a pressed juice from E. p. $(4.0 \mathrm{mg}$ cichoric acid $/ \mathrm{ml})$ or 10 20-30-40-50 ml of a homeopathic mother tincture from E.p. (containing $60 \%$ ethanol; $24.7 \mu \mathrm{g}$ alkamide- $8,9 / \mathrm{ml}$ ) were given orally as a single dose to four healthy volunteers at each dose level $(n=40)$. From each volunteer blood samples and urine were collected (blood samples: $\mathrm{t}=0,10,20,30,40,50,60,90 \mathrm{~min}, 2 \mathrm{~h}, 4 \mathrm{~h}, 8 \mathrm{~h}, 12 \mathrm{~h}, 24 \mathrm{~h}$; urine: $0 \mathrm{~h}, 0$ $24 \mathrm{~h}$ ). Cichoric acid and alkamides-8,9 were quantified in serum and urine using HPLC. From the results the pharmacokinetic parameters 
AUC, $\mathrm{C}_{\max }, \mathrm{t}_{\max }$ and $\mathrm{t}_{1 / 2}$ were calculated. Furthermore we determined immune parameters (blood counts, lymphocyte subpopulations, ex-vivo cytokine secretion in mitogen-stimulated whole blood cell cultures; $\mathrm{t}=0 \mathrm{~h}, 4 \mathrm{~h}, 8 \mathrm{~h}, 24 \mathrm{~h}$ ) and tolerability. Results: After application of the pressed juice the serum concentration of the water soluble cichoric acid increased to a maximum after 1 to $4 \mathrm{~h}$, followed by an exponential decrease with $t_{1 / 2}$ of 11.0 to $29.7 \mathrm{~h}$. Application of the homeopathic mother tincture resulted in an increase of the lipophilic alkamides-8,9 to a maximum serum concentration after 20 to $40 \mathrm{~min}$, followed by an exponential decrease with $\mathrm{t}_{1 / 2}$ of 0.3 to $2.5 \mathrm{~h}$. In contrast to cichoric acid the alkamides- 8,9 could not be detected in urine. Between the applied dose of cichoric acid rsp. alkamides- 8,9 and the pharmacokinetic parameters AUC and $\mathrm{C}_{\max }$ correlations could be observed. Effects of E.p. on the measured immune parameters could not be differentiated from influences due to the physiological circadian rhythms. After application of (unusual) high doses of both E. p. preparations, the volunteers complained about headache, tiredness and (after application of the homeopathic mother tincture) dizziness. Severe side effects were not noted. Conclusions: We are the first who describe the pharmacokinetics of the immunomodulating substances alkamides- 8,9 and cichoric acid from Echinacea purpurea after oral application. Further studies with larger sample sizes and longer periods of treatment and observation are needed to investigate potential correlations between pharmacokinetic parameters of E. p. and immunological effects.

\section{The Absorption of Enzymes from the Gut and their Effect on Tissue Repair}

Seifert, J.; Siebrecht, D.; Lange, J.P.

Experimental Surgery of the Clinic for General Surgery and Thoracic Surgery of the University of Kiel, Germany

Background and Objective: It is generally accepted that orally applied enzymes are degraded in the gut because enzymes have a protein structure. On the other hand, it has been shown that orally applied enzymes can improve wound healing and tissue repair, although enzymes are proteins and proteins are degraded in the small intestine. Therefore the absorption of 6 different enzymes (amylase, trypsin, chymotrypsin, lipase, papain, bromelain) was studied in rats with immunological as well as with isotopic methods. Methods: Antibodies against the enzymes were produced by immunization of rabbits. For the study of absorption rats (for every enzyme $n=6$ ) were fed with $10 \mathrm{mg}$ of radioactively labelled enzymes. Blood samples were taken in regular intervals over the time of 6 hours and the radioactivity measured by a szintillation counter. The undegraded macromolecular absorbed enzymes were determined by radiochromatography and immunological tests. The absorption rate could be calculated by the rest activity of the enzymes in the gut after 6 hours and the originally applied quantity. In a second investigation a traumatically induced edema of the hind leg of rats was treated by an oral application of enzyme and compared to untreated controls. Results: The concentration of the radioactively labelled enzymes in the blood showed marked and significant differences between the chosen substances. The highest concentration were found with regard to trypsin, bromelain, and amylase, whereas lipase, chymotrypsin, and papain were low concentrated in the blood. The absorption rate of trypsin was $50 \%$, of bromelain $45 \%$, of amylase $42 \%$, of chymotrypsin $38 \%$, of papain $26 \%$, and of lipase not measurable. The highest proportion of undigested high molecular enzyme in serum was found after oral amylase application (77\%), trypsin application (54\%), and bromelain $(52 \%)$, whereas all other values were below $30 \%$, and after lipase application even zero. The treatment of the edema of the hing limb of rats with bromelain reduced the edema significantly $(50 \%)$ within the first week compared to control animals. Conclusion: From this observa- tion it can be concluded that orally applied enzymes can be absorbed from the gut partially in the undegraded form and can significantly reduce inflammation and accelerate tissue repair. This investigations substantiate the fact that doctrines like the protein degeneration and absorption in the gut must be revised from time to time and that the success of complementary methods can be prooven by scientific investigations.

Interaction of Patients' Expectation on Acupuncture as an Antiemetic Adjunct Treatment

Shen, J.; Wenger, N.; Glaspy, J.; Hays, R.; Shekelle, P.

National Institutes of Health, and University of California Los Angeles, School of Medicine, USA

Background and Objectives: Most patients receiving myeloablative chemotherapy experience severe nausea and vomiting despite aggressive antiemetic pharmacotherapy. Prior studies suggested that acupuncture might be an useful adjunct in reducing chemotherapy-induced side effects. We assessed whether patients' expectation can affect symptomatic outcome in the use of acupuncture in myeloablative chemotherapy. Methods: Women receiving myeloablative chemotherapy for advanced breast cancer were randomly assigned to 3 treatment groups [1]. Specific low-frequency electro-acupuncture 'de Qi' at P-6 and ST36 for 20 minutes daily for 5 days; (2) 'nonspecific acupuncture' at control points near LU-7 and GB-34 with mock electro-stimulation on the same schedule; (3) no adjunct acupuncture. All subjects received identical four-day chemotherapy and aggressive antiemetic pharmacotherapy. The patient's expectation of treatment effect was measured at baseline prior to the start of chemotherapy. Chemotherapy-induced symptoms were self-recorded daily using a previously validated symptom diary. Results: Patients' expectations were comparable at baseline across treatment groups. For all patients, bivariate analysis of nausea by pretreatment expectation did not reveal significant correlation. A significant effect in nausea symptom was found across treatment groups; further testing found heterogeneity and confirmed an interaction effect between expectation and treatment. Conclusions: We found that patient's pretreatment expectation did not strongly impact symptom outcome as a main effect; rather, expectation exerts its influence through an interaction with the adjunct antiemetic acupuncture treatment.

\section{Reference:}

1 Cassileth BR, Lusk EJ, Bodenheimer BJ, Farber JM, Jochimsen P, Morrin-Taylor B: Chemotherapeutic toxicity - The relationship between patients' pretreatment expectations and posttreatment results. Am J Clin Oncol 1985;8(5):419-25.

\section{A Randomized Trial Comparing Acupuncture, Massage and Self-Care for Chronic Low Back Pain}

Cherkin, D. ${ }^{1}$; Eisenberg, D. ${ }^{2}$; Sherman, K. $^{3}$; Barlow, $W^{1}$; Kaptchuk, T.J. ${ }^{2}$; Street, J. ${ }^{1}$; Deyo, R. $^{4}$

${ }^{1}$ Group Health Center for Health Studies, Seattle, WA; ${ }^{2}$ Harvard Medical School, Boston, MA; ${ }^{3}$ Northwest Institute of Acupuncture, Seattle, WA; ${ }^{4}$ University of Washington, Seattle, WA, USA

Background and Objectives: This study compares the effectiveness and cost of acupuncture, therapeutic massage, and self-care educational materials for patients with chronic low back pain. Methods: Two hundred sixty-two adults between 20 and 70 years of age and with at least six weeks of low back pain were randomized to Traditional Chinese Medical $($ TCM) acupuncture $(n=94)$, soft-tissue massage $(n=78)$ or self- 
care information $(\mathrm{n}=90)$. Up to 10 treatments over 10 weeks were allowed. Symptoms, function, disability, health care utilization and cost, and satisfaction were assessed via telephone interviews at 4,10 , and 52 weeks after randomization. Results: Subjects in the massage and acupuncture groups were much more satisfied with their care than those assigned to self-care education $(\mathrm{P}<0.005)$. At 10 weeks, differences among the treatments were significant for symptoms $(P=0.015)$ and function ( $P=0.0004)$. Pairwise comparisons revealed significant differences between massage and self-care for both symptoms $(P=0.012)$ and function $(\mathrm{P}=0.0004)$ and between acupuncture and massage for function $(\mathrm{P}=0.01)$. After one year, symptoms and function were similar in the massage and self-care groups $(\mathrm{P}>0.4)$. However, the outcomes for the massage group were significantly better than for the acupuncture group for both symptoms $(P=0.002)$ and function $(P=0.05)$. Use of medication was significantly lower in the massage group than in the self-care group at both 10 weeks and one year. One year costs of back care were about $40 \%$ lower in the massage group than in the other groups $(P=0.15)$. Conclusion: These findings suggest that therapeutic massage is an effective treatment for persistent low back pain both in the short-term and long-term. TCM acupuncture appears to have relatively little effect on symptoms or function. Self-care education was relatively ineffective in the short-term but has long-term benefits similar to those of massage.

\section{Randomized Trial with Mistletoe Injections for Osteoarthritis of the Knee in Comparison to Treatment with Diclofenac}

Stange, $R^{1}{ }^{1}$; Moser, $C^{1}$; Goedings, $P^{2}$; Mansmann, $U^{3}$; Buehring, $M .{ }^{1}$ ${ }^{1}$ Klinik für Naturheilkunde, Klinikum Benjamin Franklin, Freie Universität Berlin; ${ }^{2} \mathrm{Fa}$. Helixor Heilmittel GmbH \& Co, Rosenfeld; ${ }^{3}$ Institut für Medizinische Statistik, Epidemiologie und Informatik, Klinikum Benjamin Franklin der Freien Universität Berlin, Germany

Objective: For more than 60 years, intracutaneous mistletoe injections have been used as a therapy for osteoarthritis of different locations, esp. the knee. Evaluation has only been undertaken in the form of case studies or uncontrolled trials. Therefore, we compared the therapy in a randomized trial to standard drug therapy. Materials and Methods: 100 patients with proven osteoarthritis of the knee were randomly assigned to be either given mistletoe injections (Salixor ${ }^{\circledR}$, Helixor Heilmittel, Germany) or retarded diclofenac $2 \times 75 \mathrm{mg} /$ day (Voltaren Resinat ${ }^{\circledR}$, Novartis Pharma, Switzerland). Main outcome measure after a 4week period of therapy was Lequesne's combined index of pain and function (L.I.). Both therapies were considered as equivalent if the $95 \%$ confidence interval of the ratio (mean L. I. change under mistletoe/mean L. I. change under diclofenac) was contained in the interval 0.8 to 1.2 . At the end of a 3-month follow-up period with deliberate therapies, all outcome parameters and further therapies in the meantime were listed. Results: There were significant L. I. reductions in, but no significant differences between both groups.

\begin{tabular}{|c|c|c|c|c|c|c|c|c|}
\hline & \multirow[t]{3}{*}{$\mathrm{n}$} & \multirow[t]{3}{*}{ Age } & \multirow{3}{*}{$\begin{array}{l}\text { Symp- } \\
\text { toms }\end{array}$} & \multicolumn{5}{|l|}{ L. I. } \\
\hline & & & & before & \multicolumn{2}{|c|}{$\begin{array}{l}\text { after } \\
4 \text { weeks }\end{array}$} & \multicolumn{2}{|c|}{$\begin{array}{l}\text { after } \\
3 \text { months }\end{array}$} \\
\hline & & & & mean s.d. & mean & s. d. & mean & s. d. \\
\hline Mistletoe & 50 & 61.5 & 11.1 & $9.94 \quad 3.41$ & $6.49^{*}$ & 3.60 & $6.70^{*}$ & 2.30 \\
\hline Diclofenac & 50 & 64.3 & 6.8 & $11.47 \quad 4.17$ & $7.92^{*}$ & 3.93 & $10.16^{* *}$ & 4.41 \\
\hline
\end{tabular}

Equivalence of both therapies could not be established (95\% confidence interval $0.725-1.270$ by the method of Sasabushi in favour of diclofenac). There were significantly fewer patients in the mistletoe group requiring additional therapies in the follow-up period than in the diclofenac group. Conclusion: Both treatments were associated with clinical improvements comparable to those observed in other clinical trials. The mistletoe injection therapy slightly missed the statistical criterion of equivalence. However, standard deviations of L. I. at baseline were large; a trial with larger sample size might have been able to demonstrate equivalence. Mistletoe therapy had better long-term effects, as demonstrated by fewer requirements for therapy in the follow-up period. Further studies should consider long-term performance as primary end-points.

\section{Hypericum for Premenstrual Syndrome: A Pilot Study}

\section{Stevinson, C.; Ernst, E.}

Department of Complementary Medicine, University of Exeter, UK

Background and Objective: There is currently no universally effective treatment for premenstrual syndrome (PMS). Anecdotal evidence suggests the herb Hypericum perforatum may alleviate symptoms, but there has been no clinical investigation of this. This pilot study aimed to investigate whether Hypericum perforatum could relieve symptoms of PMS in a small group of women in order to establish a hypothesis and to test methods for conducting a future randomised controlled trial (RCT). Methods: In an uncontrolled pilot study, 19 women with self-reported PMS who were in otherwise good physical and mental health and not taking other treatments for PMS underwent a preliminary screening interview, completed daily symptom ratings (DSR) for one cycle and attended a medical screening visit before being diagnosed with PMS. Participants took hypericum tablets for two complete menstrual cycles $(1 \times 300 \mathrm{mg}$ hypericum extract per day standardised to $900 \mu \mathrm{g}$ hypericin). Results: There were significant reductions on all outcome measures. The degree of improvement in overall PMS scores between baseline and the end of the trial was $51 \%$, with over two thirds of the sample demonstrating at least a $50 \%$ decrease in symptom severity. Tolerability and compliance with the treatment were encouraging. Conclusion: The results of this pilot study suggest that there is scope for conducting a randomised, placebo-controlled, double-blind trial to investigate the value of hypericum as a treatment for PMS.

\section{Acupuncture in Chronic Headache}

Stör, $W^{1}{ }^{1}$; Beyer, $A^{2}{ }^{2}$; Gleditsch, J.; Dollinger, T.; Zehender, S.; Koczorek, K.R.; Peter, K. ${ }^{2}$

${ }^{1}$ Icking, ${ }^{2}$ Klinik für Anästhesiologie, LMU, München

Objective: To observe short- and long-term effects of acupuncture in patients with chronic headache (tension type headache, migraine, cervicogenic headache, and posttraumatic headache according to definition by the IHS). Methods: We studied 12 female and 13 male patients with a history of chronic headache since $8.8 \pm 1.7$ years. We recorded levels of continuous daily pain, intensity and frequency of pain attacks, mood, depression, doctor-shopping and pain medication by means of visual analogue scales, pain diaries and questionnaires. Data were processed as comparisons of means and by analysis of individual cases to evaluate the rate of 'responders'. Results: Short-term results after 8 treatments with acupuncture once per week: intensity and frequency of pain attacks were reduced significantly as was mood state; daily continuous pain was not improved. The responder rate was $57 \%$. Long-term results at $13.1 \pm 2.3$ months: frequency of pain attacks was still clinically relevantly reduced as was doctor-shopping. Analgesic medication intake was abolished in $32 \%$ and substantially reduced in $37 \%$ of patients. Intensity of pain attacks had returned to baseline levels, as had mood 
state; continuous daily pain was still unchanged, depressive mood had deteriorated. The responder rate was $31 \%$ and $25 \%$ respectively. Conclusions: In our group of chronic pain patients acupuncture did improve frequency and intensity of pain attacks and mood state, but not continuous pain. On long-term observation, frequency of pain attacks, doctor-shopping and intake of analgesic medication remained reduced, but intensity of pain attacks and mood state had returned to baseline levels.

\section{Acupuncture versus Sumatriptan for Early Treatment of Acute Migraine Attacks - A Randomized Controlled Clinical Trial}

Melchart, D. ${ }^{1}$; Thormählen, ${ }^{1}{ }^{1}$; Hager, S. $^{2}$; Liao, ${ }^{2}{ }^{2}$; Weidenhammer, $W^{1}$

${ }^{1}$ Centre for Complementary Medicine Research, Dept. of Internal Medicine II, Technische Universität München; ${ }^{2}$ Hospital for Traditional Chinese Medicine, Kötzting, Germany

Background and Objective: While there is a number of randomized trials investigating the effectiveness of acupuncture for the prophylactic treatment of migraine, the effects of acupuncture in the treatment of acute migraine attacks have rarely been studied. We investigated whether TCM-acupuncture for early treatment of acute migraine attacks is equivalent to Sumatriptan (selective $5 \mathrm{HT}_{1}$ receptor-agonist) or superior to a placebo-medication. Methods: This trial was a randomized, partly double-blind (Sumatriptan versus placebo medication) controlled study. 179 patients experiencing the first symptoms (individually predefined prodromi) of a developing migraine attack were allocated randomly to a treatment either with acupuncture, Sumatriptan or placebo medication. Main acupuncture points were GB 20, GB 15, GB 14, GB 10, GB 8, LI 4, Liv 3, SJ 5, Du 20, Ex 2 Taiyang, completed by points for individual acompanying symptoms. Sumatriptan and placebo were applied subcutaneously. Main outcome measure was the number of patients with a fully developed migraine attack (defined as more than 30 points on a subjective rating scale by Heller) within 48 hours. Further outcome parameters were the duration and the intensity of the migraine attack as well as the number and the intensity of acompanying symptoms. Preliminary Results: Patients suffered from migraine for 21.3 years, with 6.2 attacks per month (means). 58 patients received acupuncture, 121 a drug therapy (verum or placebo). Before entering the trial 136 patients had already been treated with acupuncture (42 successfully, 27 partly successfully and 65 without success), 40 had no previous experience with acupuncture. The final results will be presented at the conference. Preliminary Conclusions: If the study shows that acupuncture is superior to placebo and maybe similarly effective as Sumatriptan, acupressure as a self-management technique for patients should be investigated in a next step.

\section{Findings in the Organo-Cutaneous Liver Zones by Connective Tissue Massage (CTM) Techniques in Women with Hormonal Inhibition of Ovulation}

\section{Uehleke, B.; Bühring, M.; Lang, A.; Hopfenmüller, W.}

Klinik für Naturheilkunde, Klinikum Benjamin Franklin, Freie Universität Berlin, Germany

Introduction: Cutaneous hyperalgia, myalgia and induration in the connective tissue of the subcutis are known as organo-cutaneous reflectory relations to malfunction of inner organs (Head, Mackenzie). CTM plays an important role in the naturopathy in Germany, but there are only few modern clinical studies available. We address the question whether a minor organic stress relates to significant findings. The meta- bolism of hormones, taken as ovulation inhibitors in women, might be interpreted as toxic stress for the liver. Methods: Without knowledge of the medical history and hormonal treatment, 100 (healthy) young women were examined manually. A skin fold at specific areas is jugded regarding volume of the skin fold $(\mathrm{V})$, induration and consistency (I), and pain $(\mathrm{P})$ after moderate pincement, and these parameters are compared to the corresponding findings at the other side of the body. After examination, medical history, including liver noxes and pharmacological treatments, was asked and documented. Women with deformation of the spine, liver-gall diseases, acute diseases, or any liver-relevant medication within the last 6 weeks are excluded. A group of healthy women with hormonal medication for at least 12 months is compared to a group of healthy women without hormonal medication. Results: There are differences between the qualities of the right zones in relation to the left body side. In the 34 women with hormonal treatment for at least 12 months, the differences are greater than in the 21 women without hormonal treatment. In 3 of the 6 investigated zones the differences between groups show statistical significance. Simultaneous consideration of all qualities and of several zones increases the significance. Discussion: A minor pharmacological stress seems to induce reflectory changes of the fibrous subcutaneous and cutaneous, or connective tissue in related liver zones of the right body side. Further studies with analogous design would be helpful for repositioning the diagnostic - and therapeutic - value of CTM.

Taking Baths: The Efficacy of Balneotherapy for Patients with Arthritis; A Systematic Review

Verhagen, A.P. ${ }^{1,2}$; de Vet, H.C.W. ${ }^{1,3}$; de Bie, R.A. ${ }^{2}$ Kessels, A.G.H. ${ }^{4}$; Boers, M. $^{5}$; Knipschild, P.G.

${ }^{1}$ Dept of Epidemiology, Maastricht University; ${ }^{2}$ Dept of General Practice, Erasmus University Rotterdam; ${ }^{3}$ Inst of Extramural Medicine, Vrije Universiteit, Amsterdam; ${ }^{4}$ Academical Medical Hospital, Maasrtricht; ${ }^{5}$ Dept of Clinical Epidemiology, Vrije Universiteit, University Hospital, Amsterdam; ${ }^{6}$ Dept of General Practice, Maastricht University, Maastricht, The Netherlands

Background and Objective: Balneotherapy (hydrotherapy or spa therapy) is one of the oldest forms of therapy for patients with arthritis. It is a very popular form of treatment for all forms of arthritis in many European countries and Israel. One of the aims of balneotherapy is to relieve the pain and to make patients feel well. We conducted a systematic review to assess the effects of balneotherapy for patients with arthritis. Studies concerned with patients with rheumatoid arthritis (RA) will form a separate group. Methods: Studies were eligible if they were randomised clinical trials and if the patients included had a form of arthritis. Balneotherapy had to be one of the interventions under study. The studies had to be published in English, German, French or Dutch. Main outcome measures included at least pain, activities of daily living or quality of life. Studies with only laboratory variables as outcome measure were excluded. The methodological quality of the studies is determined using the Delphi criteria list. Quality assessment and data extraction is carried out independently by two reviewers. Results: Ten trials with in total 607 patients are included in this review. Six studies are concerned with patients with RA. Most studies reported positive findings, but only three studies performed a between group analysis to support this claim. Often the data presentation was poor, meaning no presentation of point estimates and measures of variability. Concealment of randomisation was mentioned once, and just one of the trials included an intention-to-treat analysis. A quality of life outcome was reported in two studies. Conclusion: Although the scientific evidence is weak one cannot ignore the positive findings reported in most trials. There is however, no conclusive evidence to support these 
claims. Most methodological flaws found in the retrieved studies could be avoided in future research. New research should use subjective assessment of pain and a quality of life measure as an outcome measure. High quality research is both possible and necessary to get more insight in the treatment value of balneotherapy.

\section{Combining Qualitatitive and Quantitative Methods in Complementary Medicine Research}

Verhoef, M.J.; Hilsden, R.J.; Casebeer, A.L.; Scott, C. Department of Community Health Sciences, University of Calgary, Alberta, Canada

Background and Objectives: Qualitative and quantitative research methods have complementary strengths and weaknesses. Combining both can lead to advantages that neither approach, if used alone, could achieve. We will discuss the advantages of combining qualitative and quantitative methods and illustrate some of our points by means of a combined study of complementary therapy (CT) use among inflammatory bowel disease (IBD) patients. Advantages of Combined Research: Advantages include 1) complementarity (adding information, i. e. numbers to words and vice versa), 2) research development (one approach is used to inform and, thus, further develop the other, such as in questionnaire development), 3) increased validity, and 4) greater understanding of results (such as understanding why CTs work in addition to establishing that they work in randomized controlled trials). Often new lines of thinking (fresh perspectives or contradictions) emerge in this type of research. Example: A survey of 189 IBD patients (response rate $70 \%$ ) to assess the extent of CT use and its determinants, was followed by in-depth interviews with $14 \mathrm{CT}$ users. Combining the results of both sub-studies confirmed the main reasons for CT use (conventional treatment does not work and/or has unacceptable side effects), but provided much more insight in why and how patients made the decision to use $\mathrm{CT}$ to manage their disease. In addition the interviews gave a much better overview of the types of CTs used and the changes taking place in use. IBD patients expressed a high need for information. We used this information to develop a quantitative survey of information needs in this population. Conclusion: While many barriers to combining qualitative and quantitative methods exist (philosophical differences, high costs, inadequate researcher training and publication bias), we believe that the benefits outweigh the difficulties and that ultimately patients will benefit from increased understanding resulting from adding qualitative methods to controlled trials or quantitative methods to qualitative research.

\section{Complementary Practitioners' View of Treatment for Inflammatory Bowel Disease: An Exploratory Study}

Verhoef, M.J.; Hilsden, R.J.; Weir, V.; Liew, T.; Rapchuk, I.

Department of Community Health Sciences, University of Calgary, Alberta, Canada

Background and Objectives: A substantial number of patients with inflammatory bowel disease (IBD) use complementary medicine (CT) to manage their disease, including chiropractic and herbal therapies. Therefore, we explored whether providers of these therapies recommend these therapies for IBD patients and how they view their treatment. Methods: The sample included 66 chiropractors, 16 herbalists, 15 health food store employees/managers and 18 pharmacists. A structured questionnaire containing two patient scenarios (active ulcerative colitis and inactive Crohn's disease) was completed either by an in-person interview or by mail. The questionnaire was pretested in each sub- ject group, and the length and mode of administration was tailored to maximize the response rate within each group. Results: All chiropractors were licensed as compared to $25 \%$ of herbalists. Most respondents had seen patients with ulcerative colitis, and at least $80 \%$ of each group except pharmacists (only 10\%) would treat these patients. Almost all chiropractors used spinal manipulation, whereas herbalists used combinations of many herbs and health food store employees used a wide range of different products including aloe vera, acidophilus, vitamins and digestive enzymes. Treatment recommendations were not consistent among herbalists or health food stores. Eighty-five percent of chiropractors and $94 \%$ of pharmacists would refer these patients as compared to only $18 \%$ of herbalists. Chiropractors would refer to general practitioners and naturopaths. On a scale from 1 (very effective) to 5 (ineffective), chiropractors rated spinal manipulation as 2.7 and herbalists rated herbal treatment as 1.7 for relieving the gastrointestinal symptoms. Health food store employees viewed their products as generally free of side effects and very effective. The results with respect to the second scenario (Crohn's disease) were very similar. Conclusion: While most respondents, except pharmacists, have seen and would treat patients with IBD, chiropractors are more consistent in their approach than herbalists and health food store employees, see their treatment as only modestly effective and are more likely to engage in concomitant care.

\section{Efficacy of Massage Therapy in Chronic Pain - A Randomized Trial}

Walach, H.; Güthlin, C.; König, M.

Dept. of Environmental Medicine and Hospital Epidemiology, University Hospital Freiburg i. B., Germany

Background: Although classical massage is used widely in Germany and elsewhere for treating chronic pain conditions, there are no randomized controlled trials (RCT). Design: RCT of classical massage compared with standard medical care (SMC) in chronic pain conditions of back, neck, shoulders, head and limbs. Outcome measure: Pain rating (9-point Likert scale; predefined main outcome criterion) at pretreatment, post-treatment and 3-month follow-up, as well as pain adjective list, depression, anxiety, mood, body concept. Results: Due to political and organizational problems only 29 patients were randomized, 19 to receive massage, 10 to SMC. Pain improved significantly in both groups, but only in the massage group was it still significantly improved at follow-up. Depression and anxiety were improved significantly by both treatments, yet only in the massage group maintained at followup. Conclusion: Despite its limitation this study shows that massage can be at least as effective as standard medical care in chronic pain syndromes. Relative changes are equal, but tend to last longer and to generalize more into psychological domains.

\section{Distant Healing Improves Quality of Life in Chronically ill Patients: Results of a Waiting-List Controlled Randomized Study}

Walach, H.; Reuter, K.; Wiesendanger, H.; Werthmüller, L.

Dept. of Environmental Medicine and Hospital Epidemiology, University Hospital Freiburg i. Br., Germany

Objective: Little is known about the effects of distant healing in chronically ill patients, the population most likely to see a healer in practice. The purpose of the study was to evaluate the effects of distant healing on chronically ill patients' quality of life. Methods: Randomized, waiting-list controlled study of distant healing (anonymous, amulet, and allowing for personal contact) in chronically ill patients. Patients were randomized to either receive distant healing or to wait for 5 months. 
Outcome measure was patient reported quality of life as expressed by the sum of all MOS SF-36 items before treatment/waiting and after 5 months of treatment/waiting. Results: 60 patients were treated by various methods of distant healing over 5 months, 59 patients waited. Quality of life improved significantly $(\mathrm{p}<0.0005)$ in the treated group (10 points), while it remained stable in the control group. Positive expectation was significantly related with outcome. Conclusion: Distant healing can substantially improve quality of life in chronically ill patients who are open to this type of treatment. Expectation and positive attitudes are important factors. A future trial should research these factors in a trial with blinded and open arms.

\section{Bach-Flower Remedies are Ineffective for Test-Anxiety: Results of a Blinded, Placebo-Controlled, Randomized Trial}

Walach, H.; Rilling, C.; Engelke, $U$.

Dept. of Environmental Medicine and Hospital Epidemiology, University Hospital Freiburg i. Br., Germany

Objective: Bach-Flower remedies are a type of alternative medication which is used increasingly for self-help purposes over the counter. The purpose was to study the efficacy of a widely used combination of Bach-Flower remedies in subjects with test-anxiety. Methods: Randomized, placebo-controlled, blinded parallel group design with partial crossing over of the placebo group to remedies after the first phase. Students with test-anxiety who had two exams at least 2 weeks apart were eligible. Anxiety was measured using a standardized, validated test-anxiety questionnaire (Test Anxiety Inventory, TAI-G) 2 weeks before the first test, 1 day before test after two weeks of treatment with Bach-Flower remedies or placebo and again 2 weeks later before the second test after two more weeks of Bach-Flower remedies. Results: 55 subjects with self-reported test-anxiety out of 61 gave valid data. There was no significant difference between the groups, but a significant decrease of test-anxiety in both groups. Conclusion: Bach-Flower remedies are an effective placebo for test-anxiety, which do not have a specific effect.

\section{Disclosure of Complementary and Alternative Medicine Usage and The Doctor-Patient Relationship}

Warber, S.L.; Corage; Baden, A.; DeRoeck, M.; Cimprich, B.; Rich, D.; Ruffin, $M$.

University of Michigan, Ann Arbor, MI, USA

Background and Objective: More than $40 \%$ of Americans use complementary and alternative medicine (CAM). As many as three-fourths of CAM users do not share this information with their doctors. This reticence is not well understood. Our purpose was to investigate cancer patients' experiences with their health care providers in relation to the issue of CAM usage. Methods: We held three focus groups of cancer survivors who used CAM during their treatment. Two independent reviewers coded each focus group transcript. Quotes and themes relating to CAM usage and the doctor-patient relationship were extracted. Results: Most of the 24 participants (18 women, 6 men) were new CAM users, beginning alternative treatments after their diagnosis of cancer. The range of CAM modalities included nutritional approaches, mind-body techniques (visualization/imagery, mediation, spirituality), and manual methods (acupuncture, massage). Emergent themes relating to the relationship with their doctors included 'the good doctor', 'unpredictable physician reactions', 'doctor's lack of CAM knowledge', 'the proactive patient', 'partnership of equals', and 'wholism'. The following theory is generated from the themes and the supporting quota- tions. Patients highly value their good doctors. Disclosure of CAM usage has an unknown potential to damage to this important relationship because doctors' reactions to disclosure are unpredictable. Further, there is little utility in CAM disclosure because doctors' CAM knowledge is very limited. Instead, patients become proactive, seeking their own informational sources, such as alternative health practitioners, peer survivors, cancer-related classes, books and internet documents. Ultimately, patients desire a doctor-patient relationship that is a partnership of equals where they are respected for their knowledge and are treated as whole persons. Conclusion: This qualitative focus group data sheds light on an important enigma of the emerging field of CAM, the reluctance of patients to disclose CAM usage to their conventional physicians. Physicians can foster disclosure by behaving with respect toward patient choices, acknowledging the breadth of intervention necessary to accomplish healing, and increasing their CAM knowledge base.

\section{The Nature of Biofield Energy for Healing: A Qualitative Study}

Warber, S.L.; Kile, G.L.M.

University of Michigan Complementary and Alternative Medicine Research Center, Ann Arbor, MI, USA

Background and Objectives: Patients often use biofield energy therapies, such as Therapeutic Touch, Qigong, Reiki, and Laying-on-ofhands, as complements to conventional medicine. Biofield energy therapists claim to alter the patient's energy for the purpose of healing. This concept is neither embraced nor well understood by Western medicine. Despite research into Therapeutic Touch and Qigong, little has been done to understand the underlying principles and terminology of energy medicine. Our objective was to explore relevant concepts about the nature of energy for healing through qualitative interviews with practicing healers. Methods: We conducted content analysis of transcriptions from audio-taped, semi-structured interviews with biofield energy therapists. The subjects were purposively selected to include at least 2 therapists from key energy modalities. Results: Eleven women and 4 men were interviewed, each having 3 or more years of experience as energy healers. Modalities included Healing Touch, Reiki, Therapeutic Touch, Polarity Therapy, and Laying-on-of-hands. Seven dimensions of the nature of energy emerged. Èntities with energy' were identified, e. g., symbols, crystals, humans, mountains. 'Sources of energy' such as a supernatural being or the earth were important. Descriptive 'metaphors of energy' were expressed, e. g., energy is like electricity. Participants described different forms of 'human energy anatomy' and the aberrations that occur with illness. 'Energy movement' was variously described. Ènergy as an agent' of healing or change was another fundamental concept. Finally, 'observation or experience of energy' was accomplished with special photographic equipment or, more often, through the human body. Modes of experience include the traditional five senses, as well as, intuitive, heightened, or clairvoyant senses. Conclusion: This qualitative study illuminates seven important concepts that pertain to the nature of biofield energy: entities with energy, sources of energy, metaphors of energy, human energy anatomy, energy movement, energy as an agent, and observation/experience of energy. By studying the utterances of energy practitioners, Western researchers and practitioners can begin to understand energy healing as a common human phenomenon with many nuanced cultural descriptions. 


\section{Survey of Adverse Events Following Acupuncture (SAFA)}

White, A.R.; Ernst, E.

Department of Complementary Medicine, School of Postgraduate Medicine and Health Sciences, Exeter, UK

Background and Objective: Acupuncture is often claimed to be safe. However, adverse events have been occasionally reported, including fatal ones. The true incidence of such events can only be determined by a prospective survey. Methods: A prospective survey was undertaken in the UK from June 1998 to February 1999 involving members of medical and physiotherapy acupuncture societies. Minor events associated with acupuncture were recorded routinely each month, together with the number of acupuncture consultations. Dangerous, significantly inconvenient or unusual events were recorded individually as they occurred. Results: Interim results were analysed in November 1999, on the first 25,500 treatments given by 77 acupuncturists. 29 major events occurred including 4 episodes of loss of consciousness, one of which was accompanied by a grand mal convulsion. The most common minor events were bleeding or haematoma (3\%), aggravation of symptoms $(1 \%)$ and pain during needling $(0.9 \%)$. Adverse events can be classified into those which were the result of errors and those which were unavoidable. (Final results will be presented at the Congress.) Conclusion: Acupuncture is not free of significant adverse events. Prospective patients should be informed of the level of risk. Efforts should be made to improve the standard of acupuncture practice.

\section{Acupuncture for Tension-Type Headache: A Subject-Blind Multicenter RCT}

White, A.R. ${ }^{1}$; Resch, K.L. ${ }^{2}$; Ernst, E. ${ }^{1}$; Members of Medical Acupuncture for Tension Headache (MATH) Group

${ }^{1}$ Department of Complementary Medicine, School of Postgraduate Medicine and Health Sciences, University of Exeter, UK; ${ }^{2}$ Forschungsinstitut für Balneologie und Kurortwissenschaft, Bad Elster, Germany

Background and Objective: Acupuncture is frequently used in the management of tension headache, although trials of its effectiveness have produced contradictory results. The aim of the present study was to compare the efficacy of acupuncture with sham acupuncture in the prevention of episodic tension-type headache. Methods: Subjects with episodic tension-type headache were recruited by participating general practitioners and the research centre, and randomised to receive either genuine, brief acupuncture to between 4 and 8 points, chosen individually, or sham acupuncture with a blunted cocktail stick on bony prominences. Treatment was given weekly for 6 weeks, then monthly for 2 months. The main outcome measure was a headache diary in which number, duration and intensity of headache were recorded daily, together with analgesic consumption. Diaries were kept for 3 weeks runin, throughout the treatment period, for 3 weeks follow-up, and for a further follow-up period 3 months later. Secondary measures included General Health Questionnaire and global assessment of change. Subject-blinding was tested after the 2nd and 6th treatments by asking patients which intervention they believed they had received. Results: 50 subjects were included in the study. No significant differences were found between the changes in the two groups for any measure at any time point. There was no significant difference between the groups in their beliefs of which intervention they had received. Conclusion: This study does not provide evidence that this form of acupuncture is effective in the prevention of episodic tension-type headache.

\section{Homeopathic Medical Practice: Diagnoses and Medical Care in a Prospective Cohort Study}

Witt, C. ${ }^{1}$; Baumann, S. ; Lüdtke, R.; Chigne, $M .{ }^{1}$; Schulze, D. ${ }^{1}$; Baur, R.; Willich, S.N. ${ }^{1}$

${ }^{1}$ Institute of Social Medicine and Epidemiology, Humboldt University of Berlin, Germany

Background and Objectives: In Germany, there is a lack of large systematic studies on alternative medicine including characteristics of patients obtaining homeopathic care. The present study aims to evaluate the spectrum of diagnoses and medical care in homeopathic medical practices as prominent field of alternative medicine. Methods: In a national prospective cohort study, patients of German physicians practising individualised homeopathy were consecutively included at their initial office visit and subsequently monitored using standardised questionnaires. The physicians documented the diagnoses (ICD-9), the diagnostic procedures and all prescriptions over the subsequent 12 months. The diagnoses were documented in textform by the physicians and coded (ICD-9) by specialist of the institute. Results: 102 physicians took part in the present study. A total of 3,199 patients have been documented $(2,046$ adults [ $38 \%$ men, $72 \%$ women, $40.3 \pm 12.4$ years], 861 children [ $52 \%$ boys, $48 \%$ girls, $6.7 \pm 4$ years]). The physicians documented an average of $2.8 \pm 1.1$ diagnoses per patient. $96 \%$ of all diagnoses were chronic diseases with an average duration of $4.0 \pm 4$ years for children and $11.7 \pm 11$ years for adults. The distribution of the diseases was in the order of frequency: 13\% respiratory (ICD-9 460$519), 12 \%$ skin (680-709), 9\% muscle and skeleton system (710-739), $7 \%$ urogenital system (580-630). $21 \%$ of the disorders were coded as symptoms (e.g. headache, sleep disturbances). The 12-month follow-up demonstrates $7.3 \pm 4.8$ homeopathic prescriptions and $0.3 \pm 0.7$ non-homeopathic prescriptions per patient and only few referrals and diagnostic procedures ( $0.04 \pm 0.3$ and $0.06 \pm 0.3$, respectively, per patient). Conclusion: To our knowledge, the present study is the largest evaluation of diagnoses and therapeutic spectrum of homeopathic medicine in Germany. The data demonstrate that homeopathic treatment is seeked primarily by patients with multiple chronic disorders. Within 12 months after initiation of homeopathic therapy, the frequency of non-homeopathic medication, diagnostic procedures and referrals by the homeopathic physicians is low, indicating only little additional costs.

\section{Development of HerbMed ${ }^{\mathrm{TM}}$ - An Interactive Evidence-Based Herbal Database}

Wootton, J.C.; Soaring Bear

Alternative Medicine Foundation, Bethesda, MD, USA

Background: 1) Health care professionals need convenient access to the supporting data underlying the use of herbs for health. 2) Pharmacists and research scientists need convenient access to information on herb actions and mechanisms of action. 3) Health care consumers need responsible and reliable information about the herbal products they purchase for medicinal or preventive use. Objective: To create an interactive, electronic herbal database on the World Wide Web (WWW) providing hyperlinked access to the scientific data underlying the use of herbs for health. Methods: HerbMed ${ }^{\mathrm{TM}}$ was designed and developed by the Alternative Medicine Foundation, Inc. Bethesda, Maryland, USA. Each herb is identified by common name and Latin binomial. Information on individual herbs is sorted into clear categories. The information is as impartial and comprehensive as possible. Some selection is necessary where there is a very large amount of information. This judgment is made by the Ph. D. Research Pharmacologist, Soaring Bear, on the basis of relevance and completeness. Information on individual 
herbs is hyperlinked directly to the scientific literature using PubMed, the freely available interface for MEDLINE, and to other research resources. A search engine allows for simple searches by own choice keywords, such as disease category, or advanced boolean searches. Results: There are 6 main categories of information for each herb entry: 1) Evidence for Activity - this includes data from controlled clinical trials, observational data and case studies, and empirical knowledge from long standing folk or traditional use. 2) Warnings - links to published information on contraindications, toxic and adverse effects, and drug interactions. 3) Preparations - gives data on commercial methods of preparation and suppliers, along with information on folk methods of preparation suitable for a home or office dispensary. 4) Mixtures these may be novel commercial mixtures or blends from traditional or folk systems where the multiple components are reputed to have a synergistic effect. 5) Mechanisms of Action - the primary constituents, the biochemistry of action, and miscellaneous information on the scientific basis of action. 6) Other - for links to herb pictures, related keyword search strategies that are automatically updated from the PubMed database, conservation and cultivation issues, and related Web sites. Conclusions: HerbMed ${ }^{\mathrm{TM}}$ is a convenient and flexible resource, freely available on the WWW (http://www.amfoundation.org/herbmed.htm). It is impartial, clear, and neutral in presentation so that users can themselves determine which level of evidence they find credible or useful for research or other purposes.

\section{Systematic Review of Case Reports on Acupuncture Adverse Events in the Japanese Literature}

Yamashita, H. ${ }^{1,2,3}$; Tsukayama, $H^{2}{ }^{2}$; White, A.R. ${ }^{1}$; Ernst, E. ${ }^{1}$; Tanno, $Y^{2}$; Sugishita, $C^{3}$

${ }^{1}$ Department of Complementary Medicine, University of Exeter, UK; ${ }^{2}$ Tsukuba College of Technology Clinic, Tsukuba; ${ }^{3}$ Department of Family Nursing, University of Tokyo, Japan

Background and Objectives: Today many cases of adverse events of acupuncture treatment can be retrieved by using medical database such as MEDLINE. However, many Japanese medical journals are not listed in MEDLINE. This means that many relevant cases are not recognised internationally. Reviewing relevant articles in Japanese language is necessary in order to understand acupuncture adverse effects more fully. Methods: We searched for the relevant articles published in the period of 1987-1998 with 'Igaku Chuo Zasshi (Japana Centra Revuo Medicina) CD-ROM version' which is the database of medical journals in Japan. The Japanese keywords which meant 'acupuncture' or 'needle' were used for the search. Then case reports of adverse events were selected by title. Results: Ninety-two articles (58 full papers and 34 abstracts, duplicate reports eliminated) which reported 105 cases of suspected acupuncture adverse effects were located. The breakdown of these cases is as follows: spinal lesion (21 cases), pneumothorax (21), infection (19), foreign body in the organ (15), argyria (10), neural injury excluding spinal lesion (10) and other miscellaneous problems (11, including 2 cases of cardiac tamponade). Almost all the cases of spinal lesion, foreign body and argyria were due to permanently embedded needles, which is recognised by acupuncture societies in Japan to be a dangerous form of therapy. Of all the 92 articles, 75 had been neither listed in MEDLINE nor included in review articles. Conclusion: Most of the collected cases of adverse events are due to malpractice or negligence rather than 'adverse reactions' in the sense of adverse reactions to drugs. The present results suggest that more medical education for the therapists, more specific regulation for the treatment methods, and an effective feedback system on such events are indispensable for the safe use of acupuncture. Especially, permanently embedded needles performed by a few particular Japanese acupuncturists should be strictly prohibited.
Prenatal Treatment with Acupuncture Affects Prostaglandin Serum Level in the First Stage of Labor

Zeisler, H.; Rabl, M.; Feistauer, S.; Witt, A.; Wiltos, B.; Winzer, C.; Husslein, P. Department of Obstet \& Gynecol, University of Vienna, Austria

Objective: To evaluate the so far controversially discussed influence of prenatal acupuncture (AP) on the duration of labor and the serum levels of prostaglandin $\mathrm{E}_{2}\left(\mathrm{PGE}_{2}\right)$ at the end of the first stage of labor. Methods: Fifty-seven women with AP treatment (group A) and vaginal delivery of a singleton at full term were included in our study. The control group (group B) consisted of 63 women. The following parameters were assessed: duration of the first stage of labor, defined as the time interval between $3 \mathrm{~cm}$ of cervical dilatation and complete dilatation, duration of the second stage of labor, the use of analgesics (pethidin) during labor, administration of epidural anesthesia, and intravenous application of oxytocin during the first and second stages of labor, the serum levels of $\mathrm{PGE}_{2}$ in group A and B. Results: Median duration of the first stage of labor was significantly shortened after AP, 196 minutes vs. 321 minutes $(\mathrm{p}<0.0001)$. Median duration of the second stage of labor was 57 minutes in both cohorts. Controls received significantly more often oxytocin during the first stage of labor compared with the study group ( $85 \%$ and $15 \%$, respectively, $\mathrm{p}=0.01$ ) and during the second stage $(72 \%$ and $28 \%$, respectively, $\mathrm{p}=0.03)$. The use of epidural anesthesia and analgetics was equal in both groups. The median serum levels of $\mathrm{PGE}_{2}$ in group A and in group B were $31.4(0.07-438)$ and $6.3(0.14-32)$ $(p=0.04)$. Median duration of the first stage of labor was 226 minutes in group A (range 85-667) and 348 minutes in group B (range 67-1065) $(\mathrm{p}<0.01)$. Conclusions: Shortened first stage of labor in AP-treated women was associated with significantly elevated serum levels of $\mathrm{PGE}_{2}$.

\section{Do Physical Functional Limitations Predict Complementary and Alternative Medicine Provider Use?}

Zick, $S$.

University of Michigan, Ann Arbor, Michigan, USA

Background and Objectives: Although chronic illness is a primary reason given for visits to complementary and alternative medicine (CAM) providers, little attention has been given to the study of the various physical functional limitations (PFL) that are often the sequelae of chronic diseases among CAM users. Physical functional limitations are defined as difficulties in doing certain physical tasks in daily life such as grasping, problems walking, and bending. This study evaluated whether PFL's are a predictor for consulting a CAM provider by investigating if individuals with PFL's are more likely to consult a CAM practitioner as compared to individuals who report no PFL's. Methods: A secondary data analysis was performed using cross-sectional data from the Medical Expenditure Panel Survey (MEPS) Household Component, round one, panel three and round two, panel one. Due to the complex nature of the MEPS sampling design weighted logistic regression analysis using Stata 6.0 was performed. Results: Unadjusted analysis showed that individuals with PFL's are approximately two times as likely to visit a CAM provider compared to people who report no PFL's. After adjusting for confounders a significant interaction between level of PFL's and race appear. African-Americans do not go to CAM practitioners until their PFL's are severe, when they are over three times more likely to visit a CAM practitioner. Whites and Hispanics with PFL's are over two times more likely to visit a CAM provider compared to whites with no reported PFL's. Native Americans, Alaskan Natives, Pacific Islanders, and Asian-American PFL's are almost six times more likely to see a CAM provider compared to Whites with no reported PFL's. Conclusion: Reasons why people consult CAM providers are still uncertain. The results of this study suggest that PFL's are another reason why individuals consult a CAM provider. An individual's race or ethnicity also appears to be an important factor in determining why people visit CAM providers. Future research into the CAM practices of racial/ethnic groups appear highly warranted in light of these findings. 


\section{Author Index}

Abbott, N. 42

Ackermann, R.T. 38

Alrek, T. 38

Aune, A. 38

Baden, A. 55

Bandelin, K. 38

Barlas, P. 49

Barlow, J.H. 49

Barlow, W. 51

Barth, H. 42

Bartsch, H.H. 45, 50

Baumann, S. 56

Baur, R. 56

Baxter, G.D. $\quad 47,49$

Becker, M. 38

Beer, A.M. 38

Behrens, N. 43

Bensoussan, A. 35

Berg, J.E. 37

Beyer, A. 43, 52

Binting, S. 38

Blank, A. 39

Bœrheim, A. 38

Boers, M. 53

Brands, M. 39

Brenke, R. 47

Brinkhaus, B. 39,43

Brockow, T. 40, 40

Bühring, M. 41, 44, 52, 53

Casebeer, A.L. 54

Ceccherelli, F. 40

Cherkin, D. 51

Chigne, M. 56

Cimprich, B. 55

Claßen, K. 42

Connolly, S. 46

Corage 55

Crothers, D. 43

de Bie, R.A. 53

de Vet, H.C.W. 53

DeRoeck, M. 55

Deuse, U. 44

Deyo, R. 51

Dillner, A. 40

Dobos, G. 47

Dollinger, T. 52

Dong, H. 37

Dziambor, H. 41

Egger, M. 32

Eisenberg, D. 31, 51

Elsässer-Beile, U. 50

Engelke, U. 55

Ernst, E. $35,42,49,49,52,56,56$, 57

Feistauer, S. 57

Fiorio, R. 50

Franke, A. 40, 40, 41

Franke, K. 40

Franke, T. 41

Freivogel, K.P. 39
Gagliardi, G. 40

Gareus, I. 44

Gebauer, S. 40

Gerhard, I. 34, 39

Giron, G.P. 40

Glaspy, J. 51

Gleditsch, J. 43, 52

Gleditsch, K. 43

Goedings, P. 52

Grüber, C. 41

Güthlin, C. $36,45,54$

Hager, E.D. $\quad 41,53$

Hahn, E.G. 39,43

Häring, B. 50

Harkness, E. 42

Harrer, B. 33

Haskell, W.L. 34

Hatahet, Z. 47

Hauns, B. 50

Hays, R. 51

Heine, H. 42

Hempen, C. 39

Hennig, J. 44

Hilsden, R.J. 54, 54

Höhmann, D. 41

Hondras, M. 32

Hopfenmüller, W. 53

Høstmark, A.T. 37

Howest, S. 44

$\mathrm{Hu}, \mathrm{K} . \quad 45$

Huber, R. 42

Huether, G. 47

Hummelsberger, J. 39

Husslein, P. 57

Irnich, D. 43

Jacobs, J. 43

Janert, S. 47

Jaroslawski, K. 45

Jöckel, K.H. 48

Jonas, W.B. 46

Joos, S. 39,43

Kaptchuk, T.J. 33, 51

Kessels, A.G.H. 53

Kile, G.L.M. 55

Kirschmann, K. 44

Kleijnen, J. 32

Knipschild, P.G. $\quad 34,53$

Koczorek, K.R. 52

Kohnen, R. 39, 43

König, A. 43

König, M. 54

Krause, R. 44

Krauss, P. 43

Kuhlmann, J. 50

Lacour, M. 44, 45

Lang, A. 53

Lange, J.P. 51

Lawson, L.D. 45

Lehmann, N. 48

Leipner, J. 45
Leohnardi, H. 39

Lewin, G. 44

Lewith, G.T. 33,46

Li, P. 39

Liao, J. 53

Liew, T. 54

Linde, K. 32, 46, 46, 47

Long, A. 36, 46

Lüdtke, R. 42, 56

Lukanov, J. 38

Maier-Lenz, H. 50

Makarian, K. 47

Maluche, C. 43

Mansmann, U. 41, 52

Massing, U. 50

MATH(Medical Acupuncture for

Tension Headache)-Group 56

Maupai, N. 43

McNeill, S. 47

Melchart, D. 32, 35, 36, 46, 46, 47, 47,53

Michalsen, A. 47

Milton, G. 48

Moebus, S. 48

Molzen, H. 43

Moser, C. 52

Müller, H. 48

Mulrow, C.D. 31,38

Natalis, M. 43

Nögel, R. 39

Osborn, C.E. 49

Osowski, S. 50

Peter, K. 52

Pittler, M.H. 49, 49

Popa, G. 41

Popov, I. 44

Pratzel, H.G. 41

Rabl, M. 57

Ramirez, G. 38

Rapchuk, I. 54

Reilly, D. 31

Rein, G. 50

Reiner, L. 41

Resch, K.L. $\quad 40,41,48,56$

Reuter, K. 54

Rich, D. 55

Riesberg, A. 41

Rigoni, M.T. 40

Rilling, C. 55

Rogora, G.A. 50

Romoli, M. 50

Rostock, M. 45, 50

Ruffin, M. 55

Ruzzante, L. 40

Sagorchev, P. 38

Saller, R. 45, 47

Schildmann, J. 44

Schmidt, S. 50

Scholz, M. 46
Schön, J. 39

Schöps, P. 43

Schuck, P. 37

Schulte, A.C. 44

Schulz, K. 50

Schulze, D. 38, 56

Schumacher, M. 50

Schumann, R.R. 47

Schuppan, D. $\quad 39,43$

Scott, C. 54

Seifert, J. 51

Senn, E. 43

Seufert, J. 39

Shekelle, P. 51

Shen, J. 51

Sherman, K. 51

Siebrecht, D. 51

Soaring Bear 56

Springer, D. 43

Stange, R. 44, 52

Steger, H.G. 47

Stevinson, C. 52

Stör, W. 43, 52

Street, J. 51

Sugishita, C. 57

Tanno, Y. 57

TCM Study Group 39

ter Riet, G. 32

Thoma, D. 42

Thormählen, J. 32, 53

Tsukayama, H. 57

Uehleke, B. 44, 53

Verhagen, A.P. 53

Verhoef, M.J. 54, 54

Vickers, A. 32

Wahn, U. 41

Walach, H. $36,44,54,54,55$

Walsh, D.M. 47

Wang, Z.J. 45

Warber, S.L. 55, 55

Weatherley Jones, E. 39

Weidenhammer, W. 35, 36, 53

Weir, V. 54

Wenger, N. 51

Werner, M. 42

Werthmüller, L. 54

White, A.R. 56, 56, 57

Wiesendanger, $\mathrm{H} . \quad 54$

Wilhelmi de Toledo, F. 48

Willich, S.N. $\quad 38,46,46,56$

Wiltos, B. 57

Winzer, C. 57

Witt, A. 57

Witt, C. 38,56

Wootton, J.C. 56

Yamashita, H. 57

Zehender, S. 52

Zeisler, H. 57

Zick, S. 57

Zunder, T. $\quad 44,45$ 